\title{
On Siegel three-folds with a projective Calabi-Yau model
}

\author{
Eberhard Freitag and Riccardo Salvati Manni
}

In two recent papers we described some Siegel modular threefolds which admit a weak Calabi-Yau model. Not all of them admit a projective model. In fact, Bert van Geemen, in a private communication, pointed out a significative example which cannot admit a projective model. A weak Calabi-Yau threefold is projective if, and only if, it admits a Kaehler metric. The purpose of this paper is to exhibit criteria for the projectivity, to treat several examples, and to compute their Hodge numbers. Some of these Calabi-Yau manifolds seem to be new. We obtain a rigid Calabi-Yau manifold with Euler number 4.

\section{Introduction}

In the papers $[9,10]$ we described some Siegel modular three-folds which admit a weak Calabi-Yau model. ${ }^{1}$ Not all of them admit a projective model. In fact, Bert van Geemen, in a private communication, pointed out a significative example which cannot admit a projective model. His comment was a starting motivation for this paper. We mention that a weak CalabiYau three-fold is projective if, and only if, it admits a Kaehler metric. The purpose of this paper is to exhibit criteria for the projectivity, to treat several examples and to compute their Hodge numbers. Some of these CalabiYau manifolds seem to be new. For example, we obtain a rigid Calabi-Yau manifold with Euler number 4.

\footnotetext{
${ }^{1}$ In this paper a weak Calabi-Yau three-fold is understood as a compact complex three-fold with vanishing first Betti number and which admits an everywhere holomorphic differential form of degree 3 without zeros.
} 
Basic for our examples is a certain complete intersection $\mathcal{X}$ of four quadrics introduced in the paper [11] of van Geemen and Nygaard:

$$
\begin{aligned}
& Y_{0}^{2}=X_{0}^{2}+X_{1}^{2}+X_{2}^{2}+X_{3}^{2}, \\
& \mathcal{X}: \quad Y_{1}^{2}=X_{0}^{2}-X_{1}^{2}+X_{2}^{2}-X_{3}^{2} \text {, } \\
& Y_{2}^{2}=X_{0}^{2}+X_{1}^{2}-X_{2}^{2}-X_{3}^{2} \text {, } \\
& Y_{3}^{2}=X_{0}^{2}-X_{1}^{2}-X_{2}^{2}+X_{3}^{2} \text {. }
\end{aligned}
$$

The variety $\mathcal{X}$ has 96 isolated singularities which are ordinary double points (nodes). One of them, called the standard node, is

$$
\eta=[\sqrt{2}, 0, \sqrt{2}, 0,1,1,0,0]
$$

In the paper [7] it has been pointed out that the results of [11] imply that $\mathcal{X}$ admits a resolution that is a (projective) Calabi-Yau three-fold. The holomorphic three form without zeros (unique up to a constant) on this model is given by

$$
\frac{X_{2}^{4}}{Y_{0} Y_{1} Y_{2} Y_{3}} d\left(X_{0} / X_{2}\right) \wedge d\left(X_{1} / X_{2}\right) \wedge d\left(X_{3} / X_{2}\right) \text {. }
$$

The basic result - essentially due to van Geemen and Nygaard [11] - is the following theorem.

Theorem. The Hodge numbers of a Calabi-Yau desingularization of $\mathcal{X}$ are

$$
h_{11}=32, \quad h_{12}=0 .
$$

Hence this Calabi-Yau manifold is rigid.

We recall that the two Hodge numbers and the Euler number $e$ are related by the formula

$$
e=2\left(h_{11}-h_{12}\right),
$$

hence the Euler number for this example is $e=64$.

In $[10]$ we introduced a finite group $\mathcal{G} \subset \mathrm{GL}(8, \mathbb{C})$ which acts on $\mathcal{X}$. It is generated by the four transformations

$$
\begin{array}{rc}
U_{1}: & \left(Y_{0}, Y_{1}, Y_{3}, Y_{2}, X_{0}, X_{3}, X_{2}, X_{1}\right), \\
U_{2}: & \left(Y_{0}, Y_{3}, Y_{2}, Y_{1}, X_{0}, X_{1}, X_{3}, X_{2}\right), \\
T: & \left(Y_{0},-\mathrm{i} Y_{1}, Y_{2},-\mathrm{i} Y_{3}, X_{1}, X_{0}, X_{3}, X_{2}\right), \\
J: & \sqrt{2} \cdot\left(X_{0}, X_{1}, X_{2}, X_{3}, Y_{0} / 2, Y_{1} / 2, Y_{2} / 2, Y_{3} / 2\right) .
\end{array}
$$


This group contains the subgroup $\mathcal{Z}$ of order 4 generated by multiplication with i. The group

$$
\overline{\mathcal{G}}:=\mathcal{G} / \mathcal{Z}
$$

acts faithfully on $\mathcal{X}$. Its order is $24576=2^{13} \cdot 3$. There is a subgroup $\mathcal{H} \subset \mathcal{G}$ of index two which leaves the holomorphic three-form invariant, namely, the group generated by

$$
U_{1} U_{2}, \quad U_{1} T, \quad U_{2} T, \quad J
$$

It also contains $\mathcal{Z}$ and

$$
\overline{\mathcal{H}}:=\mathcal{H} / \mathcal{Z}
$$

is a group of order $12288=2^{12} \cdot 3$ which acts faithfully on $\mathcal{X}$ and transitively on the nodes. It leaves the holomorphic three-form invariant. In [10] the following theorem has been proved.

Theorem. Let $G$ be any subgroup of $\overline{\mathcal{H}}$. Then there exists a desingularization (in the category of complex spaces) of $\mathcal{X} / G$ that is a weak Calabi-Yau three-fold.

Actually, we proved more in [10], namely that the varieties $\mathcal{X} / G$ are Siegel three-folds.

There are 4117 conjugacy classes of subgroups of $\overline{\mathcal{H}}$ so, in principle, we get 4117 examples of weak Calabi-Yau three-folds. Of course, there might be biholomophic equivalent ones among them, but, this may be difficult to decide.

Let $G \subset \overline{\mathcal{H}}$ be a subgroup. One can ask whether there exists a resolution of $\mathcal{X} / G$ in the form of a projective Calabi-Yau manifold. In all cases that we are able to treat we will construct first a resolution $\tilde{\mathcal{X}} \rightarrow \mathcal{X}$ such that $G$ extends to a group of biholomorphic self-maps of $\tilde{\mathcal{X}}$. After that we will construct a resolution of $\tilde{\mathcal{X}} / G$ in the form of a weak Calabi-Yau manifold.

We explain the construction of $\tilde{\mathcal{X}}$. It is a mixed resolution in the sense that some of the nodes are replaced by a $\mathbb{P}^{1} \times \mathbb{P}^{1}$ (big resolution) and the rest by a $\mathbb{P}^{1}$ (small resolution). For this we have to consider two classes $\mathcal{A}$, $\mathcal{B}$ of nodes of $\mathcal{X}$.

To describe them we need the transformation

$$
g: \quad\left(Y_{0}, \ldots, X_{3}\right) \longmapsto\left(Y_{0},-Y_{1}, Y_{2},-Y_{3}, X_{0}, X_{1},-X_{2},-X_{3}\right) .
$$

It occurred already in [10]. It is a central involution of the stabilizer of the standard node. It has the following property. Consider the blow up of the node. The exceptional divisor $\left(\cong \mathbb{P}^{1} \times \mathbb{P}^{1}\right)$ is in the fixpoint locus of $g$. 
We also need the small resolutions of a node. A resolution is called small if the exceptional set is a curve. One knows that a node a admits two (isomorphism classes) of small resolutions. By a ruling of the node we understand the choice of one of the two small resolutions. Let $\overline{\mathcal{G}}_{a}$ be the stabilizer of the node $a$ with respect to the $\overline{\mathcal{G}}$-action. There is a subgroup of index two of $\overline{\mathcal{G}}_{a}$ that preserves the rulings. We described this subgroup in [10]. This subgroup extends to the small resolutions of the node. Its intersection with $\overline{\mathcal{H}}$ is a subgroup of index two of $\overline{\mathcal{H}}_{a}$.

Definition. Depending on a subgroup $G \subset \overline{\mathcal{H}}$ we define two classes $\mathcal{A}, \mathcal{B}$ of nodes of $\mathcal{X}$.

(1) A node $a$ belongs to the class $\mathcal{A}$ if its stabilizer $G_{a}$ contains an element $g$ that is conjugate to the transformation

$$
\left(Y_{0}, \ldots, X_{3}\right) \longmapsto\left(Y_{0},-Y_{1}, Y_{2},-Y_{3}, X_{0}, X_{1},-X_{2},-X_{3}\right)
$$

(2) The class $\mathcal{B}$ consists of all nodes a such that the elements of the stabilizer $G_{a}$ preserve the rulings of the node.

These two classes need not to be disjoint. Assume now that $\mathcal{A} \cup \mathcal{B}$ is the set of all nodes. Then we can construct a resolution $\tilde{\mathcal{X}} \rightarrow \mathcal{X}$ as follows. For the nodes in $\mathcal{A}$ we take the blow up (which gives the big resolution) and for the rest we take a small resolution. By definition of $\mathcal{B}$ this can be done in such a way that $G$ acts on $\tilde{\mathcal{X}}$ as group of biholomorphic transformations. Now a general theorem, essentially due to Roan ([14, Main Theorem, p. 493]), shows the following result. (compare [10, Theorem 1.5]).

Theorem. Let $G \subset \overline{\mathcal{H}}$ be a subgroup such that each node is contained in $\mathcal{A} \cup \mathcal{B}$. Then their exists a resolution $\tilde{\mathcal{X}} \rightarrow \mathcal{X}$ in the category of complex spaces such that the action of $G$ extends to $\tilde{\mathcal{X}}$ and such that $\tilde{\mathcal{X}} / G$ admits a resolution in the form of a weak Calabi-Yau three-fold. There are 2791 conjugacy classes of subgroups of $\overline{\mathcal{H}}$ with this property.

Now there arises the question whether $\tilde{\mathcal{X}}$ can be obtained as projective variety. If this is the case we can construct the resolution $\tilde{\mathcal{X}} / G$ in the form of the Hilbert scheme $G$-Hilb $(\tilde{\mathcal{X}})$ as a (projective) Calabi-Yau manifold (see [2] and [13], compare also [9, Theorem 2.6]). We will derive a projectivity criterion to obtain the following theorem. We shall reformulate it in Theorem 6.1 in a more precise way that opens a computational access. 
Theorem. Let $G \subset \overline{\mathcal{H}}$ be a group such that $\mathcal{A} \cup \mathcal{B}$ is the set of all nodes. Assume that for each node $a \in \mathcal{B}, a \notin \mathcal{A}$, there exists a $G$-invariant divisor on $\mathcal{X}$ that is not the divisor of a meromorphic function in any small (analytic) open neighborhood of a. Then $\tilde{\mathcal{X}}$ can be constructed as projective manifold. As a consequence, there exists a resolution of $\mathcal{X} / G$ in the form of a (projective) Calabi-Yau manifold. There are 1986 conjugacy classes of subgroups of $\overline{\mathcal{H}}$ with this property.

We will frequently use the following notation.

Definition. Let $D$ by a divisor on an irreducible normal complex space $X$. The divisor is called trivial at a point $a \in X$ if it is the divisor of meromorphic function in a small neighborhood of $a$.

To prove projectivity, one needs information about the map $\mathrm{Cl}(\mathcal{X}) \rightarrow$ $\mathrm{Cl}(\mathcal{X}, a)$. Here $\mathrm{Cl}(\mathcal{X})$ is the group of divisor classes of $\mathcal{X}$, and the group $\mathrm{Cl}(\mathcal{X}, a)$ is the limit of the analytic divisor class groups of small neighborhoods of $a$.

Due to the results of van Geemen and Nygaard, the group $\mathrm{Cl}(\mathcal{X})$ has rank 32. In [10], Section 2 we described an explicit system of generators of $\mathrm{Cl}(\mathcal{X}) \otimes_{\mathbb{Z}} \mathbb{Q}$. This explicit system enabled us to describe the action of $\overline{\mathcal{G}}$ on $\mathrm{Cl}(\mathcal{X}) \otimes_{\mathbb{Z}} \mathbb{Q}$. The group $\mathrm{Cl}(\mathcal{X}, a)$ is isomorphic to $\mathbb{Z}$ for a node. $\mathrm{A}$ projective small resolution of a node can be obtained if one blows up a divisor that is not trivial at the node. By the above definition this means that the image in $\mathrm{Cl}(\mathcal{X}, a)$ is different from 0 . Hence we have to describe the images of the generating divisors in $\mathrm{Cl}(\mathcal{X}, a)$. This is done in Sections 3-5 using analytic methods. We use the description of van Geemen and Nygaard's example as modular variety. The nodes correspond to certain zero-dimensional cusps. We develop a theory of local Borcherds products to describe $\mathrm{Cl}(\mathcal{X}, a)$. This theory enabled us to compute the map $\mathrm{Cl}(\mathcal{X}) \rightarrow \mathrm{Cl}(\mathcal{X}, a)$ explicitly.

After this paper has been submitted, a referee indicated a different geometric method to determine the images of the generating divisors in the local divisor class groups. Slawomir Cynk explained to us the details of this method. We added the argument after Proposition 5.6. Actually this proof is easier than our original one. Somebody who is only interested in the description of some new Calabi-Yau manifolds might prefer this proof and then he can eliminate the theory of Siegel modular varieties completely. But who is basically interested in Siegel modular varieties as the authors are, may appreciate the modular background. 
It may look rather involved to introduce a new theory of local Borcherds products in this context. But this theory may be of interest in its own right and so we kept it in this paper.

In the last three sections we show how the Hodge numbers of many examples can be computed. Many examples rest on computer calculations. Nevertheless we treated some examples in detail where the calculations can be done by hand.

We feel that it is useless to publish any programs. Some hints about them are in the text. In the Appendix at the end of the paper we give a short comprising description how the computations are organized, so anybody who is interested can control the numerical results.

\section{The divisor class group}

In the paper [10] we investigated the divisor class group $\mathrm{Cl}(\mathcal{X})$. As we mentioned, the dimension of $\mathrm{Cl}(\mathcal{X}) \otimes_{\mathbb{Z}} \mathbb{Q}$ is 32 . In [10] generators have been found using the decomposition of the divisor of Igusa's modular form $\chi_{35}$ into irreducible components. We reproduce these results in a modified form. For this we start with the three forms

$$
X_{2}-X_{3}, \quad X_{0} X_{2}+X_{1} X_{3}, \quad X_{0}-X_{1}-X_{2}-X_{3}
$$

Their zero divisors on $\mathcal{X}$ are not irreducible. Each of them can be decomposed into two divisors as follows:

$$
\begin{array}{ll}
D_{1}^{ \pm}: & X_{2}-X_{3}=Y_{1} \pm Y_{3}=0 \\
D_{2}^{ \pm}: & X_{0} X_{2}+X_{1} X_{3}=Y_{0} Y_{1} \pm Y_{2} Y_{3}=0, \\
D_{3}^{ \pm}: & X_{0}-X_{1}-X_{2}-X_{3}=Y_{1}\left(X_{1}+X_{3}\right) \pm(\sqrt{2} / 2) Y_{2} Y_{3}=0 .
\end{array}
$$

To be precise, we mention that this is only a set theoretical description. The precise definition of - for example $D_{3}^{+}$- is that the associated ideal is the radical of the image of the ideal

$$
\left(X_{0}-X_{1}-X_{2}-X_{3}, Y_{1}\left(X_{1}+X_{3}\right)+(\sqrt{2} / 2) Y_{2} Y_{3}\right)
$$

in the coordinate ring of $\mathcal{X}$ (factor ring of $\mathbb{C}\left[Y_{0}, \ldots, X_{3}\right]$ by the defining ideal). We will make use of the orbits of the divisors $D_{i}^{ \pm}$under $\mathcal{G}$.

Lemma 1.1. For every $i \in\{1,2,3\}$ the divisor $D_{i}^{+}$and $D_{i}^{-}$are in the same $\mathcal{G}$-orbit. The order of the orbit of the divisors $D_{i}^{ \pm}$under $\mathcal{G}$ is 48 for $D_{1}^{ \pm}, 12$ for $D_{2}^{ \pm}$, and 128 for $D_{3}^{ \pm}$. 
From [10] we recall the result that $\operatorname{Cl}(\mathcal{X}) \otimes_{\mathbb{Z}} \mathbb{Q}$ is the direct sum of $\mathcal{G}$-irreducible subspaces of dimension $1,3,12,16$. We consider the subspaces of $\mathrm{Cl}(\mathcal{X}) \otimes_{\mathbb{Z}} \mathbb{Q}$ generated by the orbits of $D_{i}$. Each of the three subspaces contains the trivial one-dimensional representation. The results described in [10] imply the following proposition.

Proposition 1.1. Consider the factor space of $\mathrm{Cl}(\mathcal{X}) \otimes_{\mathbb{Z}} \mathbb{Q}$ by the onedimensional representation. Its three irreducible components can be described as the $\mathcal{G}$-submodules generated by the divisors $D_{i}^{ \pm}$. More precisely, $D_{1}^{ \pm}$ generates the subspace of dimension $12, D_{2}^{ \pm}$generates the subspace of dimension 3 and $D_{3}^{ \pm}$generates the subspace of dimension 16 .

We want to describe how the generating divisors run into the standard node. For this we need information about its stabilizer. It has been described in $[10]$.

Lemma 1.2. The following transformations stabilize the standard node and preserves its ruling:

$$
\begin{aligned}
& \left(Y_{0}, Y_{3}, Y_{2}, Y_{1}, X_{0}, X_{1}, X_{3}, X_{2}\right) \\
& \left(Y_{0}, \mathrm{i} Y_{1}, Y_{2}, \mathrm{i} Y_{3}, X_{1}, X_{0}, X_{3}, X_{2}\right) \\
& \left(Y_{2}, Y_{3}, Y_{0}, Y_{1}, X_{0}, X_{1},-\mathrm{i} X_{2},-\mathrm{i} X_{3}\right), \\
& \left(Y_{0}, Y_{1}, Y_{2},-Y_{3}, X_{0}, X_{1}, X_{2},-X_{3}\right), \\
& \left(Y_{0},-Y_{1}, Y_{2},-Y_{3}, X_{0}, X_{1}, X_{2}, X_{3}\right), \\
& \left(Y_{0}, Y_{1}, Y_{2}, Y_{3}, X_{0}, X_{1},-X_{2},-X_{3}\right), \\
& \left(2 X_{0}, 2 X_{2}, 2 X_{1}, 2 X_{3}, Y_{0}, Y_{2}, Y_{1}, Y_{3}\right)
\end{aligned}
$$

Their images in $\overline{\mathcal{G}}$ generate a subgroup order $128=2^{7}$ which is a subgroup of index two of the stabilizer.

Now we consider the set of all basic divisors that run into the standard node and decompose it into orbits.

Theorem 1.1. Consider the set of 188 divisors described in Lemma 1.1. Let $\mathcal{S}$ be the subset of divisors that run into the standard node. The group of all elements of $\mathcal{G}$ that stabilize the standard node and its rulings (see Lemma 1.2 ) acts on this set. The set $\mathcal{S}$ decomposes under this group into six orbits that can be represented be the divisors

$$
D_{1}^{+}, D_{1}^{-}, D_{2}^{+}, D_{2}^{-}, D_{3}^{+}, D_{3}^{-} \text {. }
$$


In the next sections we will use this result to determine the classes of the basic divisors in small neighborhood of a node.

\section{The local divisor class group of a node}

Let $M$ be a locally compact space. An open subset $U$ is called a neighborhood of $\infty$ if its complement is a compact subset. We define

$$
H_{\infty}^{q}(M, \mathbb{Z}):=\lim _{\longleftarrow} H^{q}(U, \mathbb{Z})
$$

as the limit of the cohomology groups of all open neighborhoods of $\infty$. There is an exact sequence

$$
\cdots \longrightarrow H_{c}^{q}(M, \mathbb{Z}) \longrightarrow H^{q}(M, \mathbb{Z}) \longrightarrow H_{\infty}^{q}(M, \mathbb{Z}) \longrightarrow H_{c}^{q+1}(M, \mathbb{Z}) \longrightarrow \cdots
$$

We mention that it is sufficient that $U$ runs through a fundamental system of open neighborhoods of $\infty$.

We apply this to the tangent bundle $M$ of the sphere $S_{n}$ for $n>1$. Explicitly, this is

$$
M=\left\{(x, y) \in \mathbb{R}^{2(n+1)} ; \quad \sum x_{i}^{2}=1, \sum x_{i} y_{i}=0\right\}
$$

Since this is homotopically equivalent to the sphere, we have

$$
H^{q}(M, \mathbb{Z})= \begin{cases}\mathbb{Z} & \text { if } q=0 \text { or } q=n \\ 0 & \text { else. }\end{cases}
$$

Poincarè duality gives (for the manifold $M$ of dimension $2 n$ )

$$
H_{c}^{q}(M, \mathbb{Z})= \begin{cases}\mathbb{Z} & \text { if } q=n \text { or } q=2 n \\ 0 & \text { else }\end{cases}
$$

If $H^{q}(M, \mathbb{Z})$ is different from 0 , its generator does not have compact support. Hence $H_{c}^{q}(M, \mathbb{Z}) \rightarrow H^{q}(M, \mathbb{Z})$ is always the zero map. Now the exact sequence above shows the following:

$$
H_{\infty}^{q}(M, \mathbb{Z})= \begin{cases}\mathbb{Z} & \text { if } q \in\{0, n-1, n, 2 n-1\} \\ 0 & \text { else. }\end{cases}
$$


We consider for a positive $C$

$$
\begin{aligned}
M(C) & =\left\{(x, y) \in M ; \quad \sum y_{i}^{2}=C\right\}, \\
M(>C) & =\left\{(x, y) \in M ; \quad \sum y_{i}^{2}>C\right\} .
\end{aligned}
$$

All these sets are homotopically equivalent. Moreover, the sets $M(>C)$ define a fundamental system of open neighborhoods of $\infty$. This shows

$$
H^{q}(M(C), \mathbb{Z})=H_{\infty}^{q}(M, \mathbb{Z})
$$

There is another way to read this result. We consider the quadric

$$
Q=\left\{z \in \mathbb{C}^{n+1}, \quad \sum z_{i}^{2}=0\right\}
$$

We want to determine the cohomology of $Q-\{0\}$. This is topologically the same as $Q(C) \times \mathbb{R}_{>0}$ where

$$
Q(C)=\left\{z \in Q, \quad \sum\left|z_{i}\right|^{2}=C\right\} \quad(C>0) .
$$

Hence $Q-\{0\}$ and $Q(C)$ are homotopically equivalent. There is a map

$$
M(1) \longrightarrow Q(2), \quad(x, y) \longmapsto z:=x+\mathrm{i} y
$$

We obtain the following result.

Lemma 2.1. Let $Q \subset \mathbb{C}^{n+1}$ be the quadric defined by $\sum z_{i}^{2}=0$. Then

$$
H^{q}(Q-\{0\}, \mathbb{Z})= \begin{cases}\mathbb{Z} & \text { if } q \in\{0, n-1, n, 2 n-1\} \\ 0 & \text { else. }\end{cases}
$$

We need an analytic version $\mathrm{Cl}_{\mathrm{an}}(X)$ of the divisor class group for an irreducible normal complex space $X$. An analytic (Weil-) divisor is a formal linear combination $D=\sum_{Y} n_{Y} Y, n_{Y} \in \mathbb{Z}$, of irreducible closed complex subspaces of codimension 1 such that for each compact subset $K \subset X$ there exist only finitely many $Y$ with $Y \cap K \neq 0$ and $n_{Y} \neq 0$. Since $X$ is normal, each non-zero meromorphic function $f$ defines a divisor $(f)$, called a principal divisor. The divisor class group is the factor group of all divisors and the subgroup of all principal divisors. If $S \subset X$ is a closed analytic subspace of codimension $\geq 2$ then $\mathrm{Cl}_{\mathrm{an}}(X)=\mathrm{Cl}_{\mathrm{an}}(X-S)$. If $X$ is a projective variety then $\mathrm{Cl}_{\mathrm{an}}(X)$ and the algebraic divisor class group $\mathrm{Cl}(X)$ are naturally isomorphic. 
We need a local variant of the analytic divisor class group. Let $X$ be a normal complex space and let $a \in X$. We set

$$
\mathrm{Cl}_{\mathrm{an}}(X, a):=\underline{\lim } \mathrm{Cl}_{\mathrm{an}}(U),
$$

where $U$ runs through all connected open neighborhoods $U$ of $a$. It is of course sufficient that $U$ runs through a fundamental system of neighborhoods.

We now consider the three-dimensional node. For our purposes it will be more convenient to replace the equation $z_{1}^{1}+\cdots+z_{4}^{2}=0$ by the equivalent equation $z_{1} z_{4}=z_{2} z_{3}$.

Lemma 2.2. Let

$$
Q:=\left\{z \in \mathbb{C}^{4} ; \quad z_{1} z_{4}=z_{2} z_{3}\right\}
$$

We have

$$
\mathrm{Cl}_{\mathrm{an}}(Q, 0) \cong \mathbb{Z}
$$

A generator can be given by the divisor which is defined by $z_{1}=z_{2}=0$. Its negative can be defined by $z_{1}=z_{3}=0$. The blow ups of these two divisors give the two small resolutions.

Proof. Since $Q$ is a Cohen-Macaulay variety, the depth of the local ring $\mathcal{O}_{Q_{\mathrm{an}}, 0}$ is 3 . This implies that the cohomology $H_{\{0\}}^{q}\left(Q_{\mathrm{an}}, \mathcal{O}_{Q_{\mathrm{an}}}\right)$ with support in the origin vanishes for $q<3$ [15],

$$
H^{1}\left(Q_{\text {an }}-\{0\}, \mathcal{O}\right)=0
$$

Here $\mathcal{O}$ denotes the sheaf of analytic functions on $Q_{\text {an }}-\{0\}$. From the exponential sequence we get

$$
0 \longrightarrow H^{1}\left(Q_{\mathrm{an}}-\{0\}, \mathcal{O}^{*}\right) \longrightarrow H^{2}\left(Q_{\mathrm{an}}-\{0\}, \mathbb{Z}\right)
$$

From Lemma 2.1 we know that $H^{2}\left(Q_{\text {an }}-\{0\}\right) \cong \mathbb{Z}$. One can check that the line bundle related to the divisor $z_{1}=z_{2}=0$ goes to a generator. This 
means that we get an isomorphism

$$
H^{1}\left(Q_{\text {an }}-\{0\}, \mathcal{O}^{*}\right) \stackrel{\sim}{\longrightarrow} \mathbb{Z} .
$$

There is natural injective map

$$
\mathrm{Cl}_{\mathrm{an}}(Q-\{0\}) \longrightarrow H^{1}\left(Q_{\mathrm{an}}-\{0\}, \mathcal{O}^{*}\right) .
$$

Since $H^{1}\left(Q_{\text {an }}-\{0\}, \mathcal{O}^{*}\right)$ is generated by the image of the divisor $z_{1}=$ $z_{2}=0$, we obtain that it is an isomorphism. We can repeat the whole consideration for

$$
\left\{z \in Q ; \quad \sum\left|z_{i}\right|^{2}<\varepsilon\right\}
$$

instead of $Q$. This finishes the proof of Lemma 2.2.

\section{The modular approach}

It is necessary for us to understand the map $\mathrm{Cl}(\mathcal{X}) \rightarrow \mathrm{Cl}(\mathcal{X}, a)$ for the nodes of $\mathcal{X}$. For this we want to use the realization of $\mathcal{X}$ as Siegel three-fold and the nodes as certain zero-dimensional cusps.

Following van Geemen and Nygaard [11], we described in [10] a certain congruence subgroup $\Gamma^{\prime} \subset \operatorname{Sp}(2, \mathbb{Z})$ such that $\mathcal{X}$ is biholomorphic equivalent to the Satake compactification $\overline{\mathbb{H}_{2} / \Gamma^{\prime}}$ of $\mathbb{H}_{2} / \Gamma^{\prime}$, namely

$$
\Gamma^{\prime}=\left\{M \in \Gamma_{2}[2,4] \cap \Gamma_{2,0, \vartheta}[4] ; \quad \operatorname{det} D \equiv \pm 1 \bmod 8\right\} .
$$

For the notations we refer to [10].

The biholomorphic map $\mathcal{X} \cong \overline{\mathbb{H}}_{2} / \Gamma^{\prime}$ is given by the map that assigns the variables $Y_{0}, \ldots, Y_{3}, X_{0} \ldots, X_{3}$ to the theta functions as follows:

$$
\begin{aligned}
& Y_{0}=\vartheta\left[\begin{array}{l}
00 \\
00
\end{array}\right](Z), \quad Y_{1}=\vartheta\left[\begin{array}{l}
00 \\
10
\end{array}\right](Z), \quad Y_{2}=\vartheta\left[\begin{array}{l}
00 \\
01
\end{array}\right](Z), \quad Y_{3}=\vartheta\left[\begin{array}{l}
00 \\
11
\end{array}\right](Z), \\
& X_{0}=\vartheta\left[\begin{array}{l}
00 \\
00
\end{array}\right](2 Z), \quad X_{1}=\vartheta\left[\begin{array}{l}
10 \\
00
\end{array}\right](2 Z), \quad X_{2}=\vartheta\left[\begin{array}{l}
01 \\
00
\end{array}\right](2 Z), \quad X_{3}=\vartheta\left[\begin{array}{l}
11 \\
00
\end{array}\right](2 Z) .
\end{aligned}
$$

The nodes correspond to certain zero-dimensional boundary components. Unfortunately to the zero-dimensional standard boundary point does not correspond a node, hence we have to change coordinates.

Lemma 3.1. The standard node corresponds to the boundary point

$$
\left(\begin{array}{cc}
0 & 0 \\
0 & \mathrm{i} \infty
\end{array}\right):=\lim _{t \rightarrow+\infty}\left(\begin{array}{cc}
\mathrm{i} / t & 0 \\
0 & \mathrm{i} t
\end{array}\right) .
$$


Proof. We can use the involution

$$
N:=\left(\begin{array}{cccc}
0 & 0 & 1 & 0 \\
0 & 1 & 0 & 0 \\
-1 & 0 & 0 & 0 \\
0 & 0 & 0 & 1
\end{array}\right)
$$

to transform this boundary point to the standard boundary point

$$
\left(\begin{array}{cc}
\mathrm{i} \infty & 0 \\
0 & \mathrm{i} \infty
\end{array}\right):=\lim _{t \rightarrow+\infty}\left(\begin{array}{cc}
\mathrm{i} t & 0 \\
0 & \mathrm{it}
\end{array}\right) .
$$

We have to transform the modular forms $Y_{0}, \ldots, X_{3}$ by means of the transformation

$$
f \mid N(Z)=\operatorname{det}(C Z+D)^{-1 / 2} f(N Z) .
$$

Standard theta transformation formulas show that the transformed forms up to a common constant factor are

$$
Y_{0}^{\prime}:=\vartheta\left[\begin{array}{c}
00 \\
00
\end{array}\right](Z), \quad Y_{1}^{\prime}:=\vartheta\left[\begin{array}{l}
10 \\
00
\end{array}\right](Z), \quad Y_{2}^{\prime}:=\vartheta\left[\begin{array}{l}
00 \\
01
\end{array}\right](Z), \quad Y_{3}^{\prime}:=\vartheta\left[\begin{array}{l}
10 \\
01
\end{array}\right](Z)
$$

and

$$
\begin{aligned}
& X_{0}^{\prime}=\frac{1}{\sqrt{2}}\left(\vartheta\left[\begin{array}{l}
00 \\
00
\end{array}\right](2 Z)+\vartheta\left[\begin{array}{l}
10 \\
00
\end{array}\right](2 Z)\right), \\
& X_{1}^{\prime}=\frac{1}{\sqrt{2}}\left(\vartheta\left[\begin{array}{l}
00 \\
00
\end{array}\right](2 Z)-\vartheta\left[\begin{array}{l}
10 \\
00
\end{array}\right](2 Z)\right), \\
& X_{2}^{\prime}=\frac{1}{\sqrt{2}}\left(\vartheta\left[\begin{array}{l}
01 \\
00
\end{array}\right](2 Z)+\vartheta\left[\begin{array}{l}
11 \\
00
\end{array}\right](2 Z)\right), \\
& X_{3}^{\prime}=\frac{1}{\sqrt{2}}\left(\vartheta\left[\begin{array}{l}
01 \\
00
\end{array}\right](2 Z)-\vartheta\left[\begin{array}{l}
11 \\
00
\end{array}\right](2 Z)\right) .
\end{aligned}
$$

The projective coordinates of the cusp $\left(\begin{array}{cc}0 & 0 \\ 0 & \mathrm{i} \infty\end{array}\right)$ with respect to the variables $Y_{0}, \ldots, X_{3}$ are the same as the coordinates of the transformed cusp $\left(\begin{array}{cc}\mathrm{i} \infty & 0 \\ 0 & \mathrm{i} \infty\end{array}\right)$ with respect to the new variables $Y_{0}^{\prime}, \ldots, X_{3}^{\prime}$. Using the formula

$$
\lim _{t \rightarrow \infty} \vartheta\left[\begin{array}{l}
a_{1} a_{2} \\
b_{1} b_{2}
\end{array}\right]\left(\begin{array}{cc}
\text { it } & 0 \\
0 & \text { it }
\end{array}\right)= \begin{cases}1 & \text { if } a_{1}=a_{2}=0 \\
0 & \text { else }\end{cases}
$$

we get for the value $[1,0,1,0,1 / \sqrt{2}, 1 / \sqrt{2}, 0,0]$. This is the standard node as claimed in Lemma 3.1 . 
We denote the conjugated group of $\Gamma^{\prime}$ by

$$
\Gamma^{\prime \prime}:=N \Gamma^{\prime} N^{-1} \text {. }
$$

The transformed forms $Y_{0}^{\prime}, \ldots, X_{3}^{\prime}$ are generators of the ring of modular forms of $\Gamma^{\prime \prime}$ [10, Proposition 2.2]. Of course, they satisfy the same relations as the $Y_{0}, \ldots, X_{3}$. One gets

$$
\begin{aligned}
\Gamma^{\prime \prime}=\{ & M \in \Gamma_{2}[2,4] ; \quad a_{12} \equiv d_{21} \equiv 0 \bmod 4, \quad b_{11} \equiv c_{22} \equiv 0 \bmod 8, \\
& \left.a_{11} d_{22}-b_{12} c_{21} \equiv \pm 1 \bmod 8\right\}
\end{aligned}
$$

The Siegel-parabolic subgroup $\mathcal{P}$ of $\Gamma^{\prime \prime}$ (defined by $C=0$ ) consists of all

$$
\left(\begin{array}{cc}
E & T \\
0 & E
\end{array}\right)\left(\begin{array}{cc}
{ }^{\mathrm{t}} U & 0 \\
0 & U^{-1}
\end{array}\right), \quad U \in \mathcal{U}, T \in \mathcal{T}
$$

Here $\mathcal{T}$ denotes the set of all integral matrices $T=\left(\begin{array}{cc}t_{0} & t_{1} \\ t_{1} & t_{2}\end{array}\right)$ that satisfy

$$
t_{0} \equiv 0 \bmod 8, \quad t_{1} \equiv 0 \bmod 2, \quad t_{2} \equiv 0 \bmod 4
$$

and $\mathcal{U}$ is the subgroup of $\operatorname{GL}(2, \mathbb{Z})$ defined by the congruences

$$
b \equiv 0 \bmod 2, \quad c \equiv 0 \bmod 4, \quad U=\left(\begin{array}{ll}
a & b \\
c & d
\end{array}\right) .
$$

This group contains non-trivial elements of finite order, for example the diagonal matrix with entries $1,-1$.

So we have proved the following result.

Lemma 3.2. There is a biholomorphic map between $\mathcal{X}$ and $\overline{\mathbb{H}_{2} / \Gamma^{\prime \prime}}$. It is defined through the correspondence $X_{i} \leftrightarrow X_{i}^{\prime}, Y_{i} \leftrightarrow Y_{i}^{\prime}$. The standard node corresponds to the standard cusp $\left(\begin{array}{c}\mathrm{i} \infty \\ 0 \\ 0\end{array}\right)$. The corresponding parabolic group is the group $\mathcal{P}$ described above.

\section{Zero-dimensional cusps}

We consider the Siegel parabolic group that consists of all integral symplectic matrices of genus two

$$
M=\left(\begin{array}{cc}
A & B \\
C & D
\end{array}\right), \quad C=0
$$


Let $\mathcal{P}$ be a subgroup of finite index. For simplicity we assume that $\mathcal{P}$ splits. This means the following. If $M$ is in $\mathcal{P}$ then the matrix

$$
M=\left(\begin{array}{cc}
A & 0 \\
0 & D
\end{array}\right)
$$

is in $\mathcal{P}$ too. Let $\mathcal{U} \subset \mathrm{GL}(2, \mathbb{Z})$ be the subgroup of all $U$ such that

$$
\left(\begin{array}{cc}
{ }^{t} U & 0 \\
0 & U^{-1}
\end{array}\right) \in \mathcal{P}
$$

and let $\mathcal{T}$ be the set of all integral symmetric matrices $T$ such that

$$
\left(\begin{array}{cc}
E & T \\
0 & E
\end{array}\right) \in \mathcal{P}
$$

Then $\mathcal{P}$ consists of all

$$
\left(\begin{array}{cc}
E & T \\
0 & E
\end{array}\right)\left(\begin{array}{cc}
{ }^{t} U & 0 \\
0 & U^{-1}
\end{array}\right), \quad U \in \mathcal{U}, T \in \mathcal{T} .
$$

The group $\mathcal{U}$ acts on $\mathcal{T}$. For $U \in \mathcal{U}$ and $T \in \mathcal{T}$ we have $T[U]:={ }^{\mathrm{t}} U T U \in \mathcal{T}$. The group $\mathcal{U}$ also acts on the dual lattice $\mathcal{T}^{*}$ that consists of all symmetric rational matrices $H$ such that $\sigma(T H) \in \mathbb{Z}$ for all $T \in \mathcal{T}$. This action is given by $T\left[{ }^{\mathrm{t}} U\right]$. Here $\sigma$ denotes the trace.

Let $Y$ be a symmetric positive definite matrix. Its minimum $m(Y)$ is defined to be the minimum value of all $Y[g]$ where $g$ runs through all nonzero integral columns. This is a continuous function on the space of all positive definite symmetric matrices. For $C \geq 0$ we denote by $\mathbb{H}_{2}(C)$ the set of all symmetric complex matrices $Z$ with positive definite imaginary part $Y$ such that $m(Y)>C$. This is an open subset of the set of all symmetric matrices. The case $C=0$ is the Siegel upper half-plane $\mathbb{H}_{2}$. The group $\mathcal{P}$ acts on $\mathbb{H}_{2}(C)$ through

$$
\left(\begin{array}{cc}
E & T \\
0 & E
\end{array}\right)\left(\begin{array}{cc}
{ }^{t} U & 0 \\
0 & U^{-1}
\end{array}\right)(Z)=Z[U]+T .
$$

Since $\mathcal{P}$ acts properly discontinuously, the quotient

$$
U_{C}=\mathbb{H}_{2}(C) / \mathcal{P}
$$

is a normal complex space. 
We are interested in the group

$$
\mathrm{Cl}(\mathcal{P}):=\lim _{C \rightarrow \infty} \mathrm{Cl}\left(U_{C}\right)
$$

This group may be very big and we are only interested in a small part of it, the Heegner part:

Let $S$ be a fixed matrix in $\mathcal{T}^{*}$ with negative determinant. For any real number $d$,

$$
\left\{Z \in \mathbb{H}_{2} ; \quad \sigma(Z S)=d\right\}
$$

is a non-empty set of codimension 1 . We denote by $H(S, d)$ the set of all $Z$ with

$$
\sigma(Z S[U]) \equiv d \bmod 1 \quad \text { for some } U \in \mathcal{U} \text {. }
$$

This can be considered as a $\mathcal{P}$-invariant divisor where the multiplicities are taken to be 1 . The matrix $S$ is called primitive if it cannot be written in the form $S=t S_{1}$ where $t>1$ is a natural number different from 1 and $S_{1} \in \mathcal{T}^{*}$. Then the equation $\sigma(S T)=1$ has a solution $T \in \mathcal{T}$. Hence, in the primitive case, $H(S, d)$ is the $\mathcal{P}$-orbit of the divisor $\sigma(Z S)=d$. In the general case, it is a finite union of such $\mathcal{P}$-orbits. This follows from the trivial formula

$$
H(N S, d)=\bigcup_{\nu=1}^{N} H\left(S, \frac{d+\nu-1}{N}\right) \quad(S \text { primitive, } N \in \mathbb{N}) .
$$

We denote the subgroup of $\mathrm{Cl}\left(U_{C}\right)$ spanned by these divisors by $\mathrm{Cl}_{\mathrm{Heeg}}\left(U_{C}\right)$. This part can be described by local Borcherds products. They have been treated in the literature in different contexts. In [1], the local divisor class group of a generic point of a one-dimensional boundary component has been treated, even more general in the context of the group $O(2, n)$. Another case that has been treated by Bruinier are the cusps of Hilbert modular surfaces in [4]. Here we have to consider the zero-dimensional cusps of Siegel threefolds.

The convergence of the local Borcherds products simply will rest on the following result, in which we use the notation $e(Z)=\exp 2 \pi \mathrm{i} \sigma(Z)$.

Lemma 4.1. Let $n>0$ be a natural number. The series

$$
\sum_{H} e(-H Y), \quad Y \text { symmetric positive definite, }
$$

converges. Here $H$ runs through all integral matrices with determinant $\operatorname{det} H=-n$ and such that $\sigma(H) \geq 0$. 
The proof is left to the reader.

We want to construct a holomorphic function with divisor $H(S, d)$. This can be done through the local Borcherds product

$$
B(Z)=\prod_{H}\left(\mathrm{e}^{2 \pi \mathrm{i} \epsilon(H)(\sigma(H Z)-d)}-1\right) .
$$

Here $H$ runs through all matrices of the form $H=S\left[{ }^{t} U\right]$ with $U \in \mathcal{U}$ and

$$
H)= \begin{cases}+1 & \text { if } \sigma(H) \geq 0 \\ -1 & \text { else }\end{cases}
$$

The sign $\epsilon(H)$ is necessary for the convergence of product. Actually Lemma 4.1 implies that - with this choice of the sign - the product converges in $\mathbb{H}_{2}$ and defines an analytic function there. Due to the sign $\epsilon(H)$ the product is not invariant under the group $\mathcal{P}$. The failure of the invariance will enable us to compute the cohomology class of the divisor $H(S, d)$ in the local divisor class group. We refer to [1] and to the article of Bruinier in [4] for similar constructions.

We have to determine the multiplicity of the local Borcherds product.

Lemma 4.2. Let $B(Z)$ be the local Borcherds product with zero set $H(S, d)$. Assume that $H(S, d)$ is in the ramification of the map $\mathbb{H}_{2}(C) / \mathcal{T} \rightarrow$ $\mathbb{H}_{2}(C) / \mathcal{P}$. Then the multiplicity of the zeros of $B(Z)$ is two. Otherwise it is one. Hence the zero order equals the ramification order.

Proof. Assume that the set $\sigma(S Z)=d$ is in the ramification. This means that there exists a substitution $Z \mapsto Z[U]+H$ in $\mathcal{P}$ that fixes it. This transformation must be of finite order, and hence $U$ is of finite order. The set

$$
\{Y>0 ; \quad \sigma(S Y)=0\}
$$

is not empty and two dimensional. The equation $Y[U]=Y$ holds on this set. We claim that $\operatorname{det} U=-1$. This follows from the fact that an element from $\mathrm{SL}(2, \mathbb{Z})$ has at most one fixed point with respect to the standard action of $\mathrm{SL}(2, \mathbb{Z})$ on the upper half-plane. The same argument shows $U^{2}= \pm E$. The minus sign is not possible, since otherwise the two eigenvalues of $U$ would be equal (both $\pm \mathrm{i}$ ) and then $U$ would be a multiple of the unit matrix. The known list of the elements of finite order in $\operatorname{GL}(2, \mathbb{Z})$ shows that each of the 
matrices $U$ is conjugate to one of the following two:

$$
U_{1}=\left(\begin{array}{cc}
1 & 0 \\
0 & -1
\end{array}\right), \quad U_{2}=\left(\begin{array}{ll}
0 & 1 \\
1 & 0
\end{array}\right)
$$

In the first case the solutions of $\sigma\left(Y\left[U_{1}\right]\right)=Y$ are the diagonal matrices. The corresponding matrix $S$ must be a constant multiple of $U_{2}$. Analogously, the matrix $S$ that corresponds to $U_{2}$ is a constant multiple of $U_{1}$. In both cases we have $S\left[{ }^{\mathrm{t}} U\right]=-S$. Hence this holds in general. We also see that the ramification order is two.

We know from the definition of $S, H$ at the beginning of the proof that

$$
\sigma(S Z)=d \Longrightarrow \sigma(S(Z[U]+H))=d
$$

Hence we get

$$
\sigma(S Z)=d \Longrightarrow \sigma(S Z)=\sigma(S H)-d .
$$

This gives $2 d=\sigma(S H) \in \mathbb{Z}$. Since in the Borcherds product besides $H=S$ also $H=-S=S\left[{ }^{\mathrm{t}} U\right]$ occurs, we obtain that the set $\sigma(S Z)=d$ is an at least double zero. It is easy to see that the multiplicity is really 2 .

The function $B$ is periodic with respect to $\mathcal{T}$. We have to study how it transforms under $\mathcal{U}$. For $U \in \mathcal{U}$ we have that

$$
J(U, Z):=B(Z[U]) / B(Z)
$$

is a cocycle,

$$
J(U V, Z)=J(V, Z[U]) J(U, Z) .
$$

(As in group cohomology usual, we consider the action of $\mathcal{U}$ from the left. This is given by $(U, f) \longmapsto g$ where $g(Z)=Z[U]$.) So $J(U, Z)$ represents an element of

$$
H^{1}\left(\mathcal{U}, \mathcal{O}^{*}\left(\mathbb{H}_{2}(C) / \mathcal{T}\right)\right)
$$

We denote the part of this group that is generated by the $J(U, Z)$ above by

$$
H_{\text {Heeg }}^{1}\left(\mathcal{U}, \mathcal{O}^{*}\left(\mathbb{H}_{2}(C) / \mathcal{T}\right)\right) \text {. }
$$

Let $B(Z)$ be a local Borcherds product. Its zero divisor, considered on $\mathbb{H}_{2}(C)$, is invariant under $\mathcal{P}$. Hence it induces a divisor on $U_{C}$. By definition, the multiplicity of a component is the multiplicity of $B$ considered on $\mathbb{H}_{2}(C)$ divided by the ramification order (which is 1 or 2). By Lemma 4.2 this quotient is an integer. Hence we obtain the following result. 
Lemma 4.3. There is a homomorphism

$$
H_{\text {Heeg }}^{1}\left(\mathcal{U}, \mathcal{O}^{*}\left(\mathbb{H}_{2}(C) / \mathcal{T}\right)\right) \longrightarrow \mathrm{Cl}\left(U_{C}\right)
$$

that attaches to the class of the cocycle $B(Z[U]) / B(Z)$ the induced divisor on $U_{C}$ with multiplicities as described above.

If we write $B(Z[U])$ and $B(Z)$ as infinite products, then in the quotient $B(Z[U]) / B(Z)$ all factors with the property $\epsilon(H) \neq \epsilon(H[U])$ cancel. The following lemma shows that there are only finitely many $H$ which do not have this property.

Lemma 4.4. Let $U \in \mathrm{GL}(2, \mathbb{Z})$ and $n>0$ a natural number. There exist only finitely many symmetric integral $H$ with the properties

$$
\operatorname{det} H=-n, \quad \epsilon(H) \neq \epsilon(H[U]) .
$$

The proof is left to the reader.

The lemma implies that the automorphy factor $J(U, Z)=B(Z[U]) /$ $B(Z)$ is of a very simple form. It is a finite product of quotients of the type

$$
\frac{\mathrm{e}^{x}-1}{\mathrm{e}^{-x}-1}=-\mathrm{e}^{x}
$$

Hence we see: the cocycle related to a local Borcherds product is of the simple form

$$
J(U, Z)=C_{U} e\left(Z H_{U}\right)
$$

where $C_{U}$ is a constant of absolut value 1 and $H_{U} \in \mathcal{T}^{*}$. These considerations give the following explicit formula for the cocycle.

Lemma 4.5. The cocycle of the local Borcherds product

$$
B(Z)=\prod_{H}\left(\mathrm{e}^{2 \pi \mathrm{i} \epsilon(H)(\sigma(H Z)-d)}-1\right)
$$

associated to $H(S, d)$ is of the form

$$
J(U, Z):=B(Z[U]) / B(Z)=C_{U} \mathrm{e}^{2 \pi \mathrm{i} \sigma\left(Z H_{U}\right)} .
$$

If $\mathcal{H}$ denotes the (finite) set of all $H=S\left[{ }^{t} V\right], V \in \mathcal{U}$, such that $\epsilon(H) \neq$ $\epsilon\left(H\left[{ }^{\mathrm{t}} U^{-1}\right]\right)$, then

$$
H_{U}=-\sum_{H \in \mathcal{H}} \epsilon(H) H
$$


and

$$
C_{U}=(-1)^{\# \mathcal{H}} \exp \left(2 \pi \mathrm{i} d \sum_{H \in \mathcal{H}} \epsilon(H)\right)
$$

We call the $\mathcal{U}$-module of the functions

$$
C e(Z H) ; \quad|C|=1, \quad H \in \mathcal{T}^{*},
$$

by $\mathcal{E}$. Then our cocycles are in the image of the natural map

$$
H^{1}(\mathcal{U}, \mathcal{E}) \longrightarrow H^{1}\left(\mathcal{U}, \mathcal{O}^{*}\left(\mathbb{H}_{2}(C) / \mathcal{T}\right)\right)
$$

Remark. We also can consider the submodule $\mathcal{E}_{0} \subset \mathcal{E}$ of all elements with $C=1$. Then $\mathcal{E}_{0} \otimes_{\mathbb{Z}} \mathbb{R}$ is isomorphic to $\operatorname{Sym}^{2}\left(\mathbb{R}^{2}\right)$. Hence there is a link to the Eichler cohomology group $H^{1}\left(\mathcal{U}, \operatorname{Sym}^{2}\left(\mathbb{R}^{2}\right)\right)$ and from there are relations to elliptic cusp forms of weight 4 . This might be an interesting project for further research.

We denote the part of $H^{1}(\mathcal{U}, \mathcal{E})$ coming from local Borcherds products by $H_{\text {Heeg }}^{1}(\mathcal{U}, \mathcal{E})$. We have proved the following result.

Lemma 4.6. There is a natural homomorphism

$$
H_{\text {Heeg }}^{1}(\mathcal{U}, \mathcal{E}) \longrightarrow \mathrm{Cl}\left(U_{C}\right)
$$

\section{A very particular case}

We take for $\mathcal{P}$ the parabolic group described in Section 3.

Lemma 5.1. The group $\mathcal{U}$ can be generated by the matrices

$$
V_{0}=\left(\begin{array}{cc}
1 & 0 \\
0 & -1
\end{array}\right), \quad V_{1}=\left(\begin{array}{ll}
1 & 2 \\
0 & 1
\end{array}\right), \quad V_{2}=\left(\begin{array}{ll}
1 & 0 \\
4 & 1
\end{array}\right), \quad V_{3}=\left(\begin{array}{ll}
3 & 2 \\
4 & 3
\end{array}\right)
$$

We skip the proof and simply mention that the group $\mathcal{U} \cap \mathrm{SL}(2, \mathbb{Z})$ is conjugate to the group $\Gamma_{0}[8]$. One can use the program MAGMA [3] to get generators for the standard subgroups of the elliptic modular group.

Theorem 5.1. In the special case of $\mathcal{P}$ above, we have

$$
\lim _{C \rightarrow \infty} \operatorname{Cl}\left(U_{C}\right) \cong \mathbb{Z}
$$


Proof. Recall (Section 2) that there exists a subgroup $\Gamma^{\prime \prime} \subset \operatorname{Sp}(2, \mathbb{Z})$ of finite index such that $\mathcal{P}$ is just the parabolic subgroup defined by $C=0$. We use some details of the Satake compactification $X_{\Gamma}^{\prime \prime}=\mathbb{H}_{2}^{*} / \Gamma^{\prime \prime}$ as described in [8] for example. For $C$ large enough the natural map

$$
\mathbb{H}_{2}(C) / \mathcal{P} \longrightarrow \mathbb{H}_{2} / \Gamma^{\prime \prime}
$$

is an open embedding. Hence we can consider then $U_{C}$ as a subset of $\mathbb{H}_{2} / \Gamma^{\prime \prime}$. There exists a fundamental system of open neighborhoods $\hat{U}_{C}$ of the standard zero-dimensional boundary component $\infty$ in $X_{\Gamma^{\prime \prime}}$ such that

$$
\hat{U}_{C} \cap\left(\mathbb{H}_{2} / \Gamma^{\prime \prime}\right)=U_{C} \quad(C \gg 0) .
$$

The complement of $U_{C}$ in $\hat{U}_{C}$ is a curve. From [10] we know that the germ $\left(X_{\Gamma}^{\prime \prime}, \infty\right)$ is isomorphic to the germ of a quadric at the origin. In [10] we have taken the quadric $Q$ defined by $z_{1} z_{4}=z_{2} z_{3}$. Now we go back to $\hat{U}(C)$. The groups $\mathrm{Cl}\left(U_{C}\right)$ and $\mathrm{Cl}\left(\hat{U}_{C}-\{\infty\}\right)$ agree, since divisors can be extended over codimension $\geq 2$. Now we can apply Lemma 2.2 to complete the proof.

We are interested in some very simple divisors, namely those which correspond to the divisors $D_{i}^{ \pm}$in Section 2. Using the coordinates described in Lemma 3.2, we have the following result.

Lemma 5.2. The equations of the transformed divisors $D_{i}^{ \pm}$in the model $\overline{\mathbb{H}_{2} / \Gamma^{\prime \prime}}$ are (set theoretically):

$$
\begin{aligned}
D_{1}^{ \pm}: & \vartheta\left[\begin{array}{l}
11 \\
00
\end{array}\right](2 Z)=\vartheta\left[\begin{array}{l}
10 \\
00
\end{array}\right](Z) \pm \vartheta\left[\begin{array}{l}
10 \\
01
\end{array}\right](Z)=0 \\
D_{2}^{ \pm}: & \vartheta\left[\begin{array}{l}
01 \\
00
\end{array}\right](Z)^{2}=\vartheta\left[\begin{array}{l}
00 \\
00
\end{array}\right](Z) \vartheta\left[\begin{array}{l}
10 \\
00
\end{array}\right](Z) \pm \vartheta\left[\begin{array}{l}
00 \\
01
\end{array}\right](Z) \vartheta\left[\begin{array}{l}
10 \\
01
\end{array}\right](Z)=0 \\
D_{3}^{ \pm}: & \vartheta\left[\begin{array}{l}
10 \\
00
\end{array}\right](2 Z)-\vartheta\left[\begin{array}{l}
01 \\
00
\end{array}\right](2 Z) \\
& =\vartheta\left[\begin{array}{l}
10 \\
00
\end{array}\right](Z) \vartheta\left[\begin{array}{l}
00 \\
10
\end{array}\right](Z / 2) \pm \vartheta\left[\begin{array}{l}
00 \\
01
\end{array}\right](Z) \vartheta\left[\begin{array}{l}
10 \\
01
\end{array}\right](Z)=0 .
\end{aligned}
$$

The proof is a straightforward calculation. We only mention that the theta relations

$$
\begin{gathered}
\vartheta\left[\begin{array}{l}
a \\
b
\end{array}\right](Z)^{2}=\sum_{x}(-1)^{\mathrm{t} x b} \vartheta\left[\begin{array}{c}
a+x \\
0
\end{array}\right](2 Z) \vartheta\left[\begin{array}{l}
x \\
0
\end{array}\right](2 Z) \\
\vartheta\left[\begin{array}{l}
00 \\
10
\end{array}\right](Z / 2)=\vartheta\left[\begin{array}{l}
00 \\
00
\end{array}\right](2 Z)-\vartheta\left[\begin{array}{l}
10 \\
00
\end{array}\right](2 Z)+\vartheta\left[\begin{array}{l}
01 \\
00
\end{array}\right](2 Z)-\vartheta\left[\begin{array}{l}
11 \\
00
\end{array}\right]
\end{gathered}
$$

have been used. 
We also can give the equations in the Siegel upper half-plane.

Lemma 5.3. Close to the standard boundary point, the divisors $D_{1}^{+}, \ldots$, $D_{3}^{-}$can be defined as images of the following sets:

$$
\begin{aligned}
& D_{1}^{+}: \quad 2 z_{1}+4 z_{2}=1, \quad D_{1}^{-}: \quad 2 z_{1}=1, \\
& D_{2}^{+}: \quad z_{0}+3 z_{1}+2 z_{2}=1, \quad D_{2}^{-}: \quad z_{0}+z_{1}=1 \text {, } \\
& D_{3}^{+}: z_{2}=z_{0}+2, \quad D_{3}^{-}: \quad z_{2}=z_{0} \text {. }
\end{aligned}
$$

Proof. We start with the divisor $D_{1}^{-}$: We restrict the series

$$
\begin{aligned}
& \vartheta\left[\begin{array}{l}
a_{1} a_{2} \\
b_{1} b_{2}
\end{array}\right](2 Z) \\
& \quad=\sum(-1)^{b_{1} g_{1}+b_{2} g_{2}} \mathrm{e}^{2 \pi \mathrm{i}\left(z_{0}\left(g_{1}+a_{1} / 2\right)^{2}+2 z_{1}\left(g_{1}+a_{1} / 2\right)\left(g_{2}+a_{2} / 2\right)+z_{2}\left(g_{2}+a_{2} / 2\right)^{2}\right)}
\end{aligned}
$$

to $2 z_{1}=1$. Since $2 g_{1} g_{2}$ is even we get

$$
\mathrm{e}^{\pi \mathrm{i} a_{1} a_{2} / 2} \vartheta\left[\begin{array}{c}
a_{1} \\
b_{1}+a_{2}
\end{array}\right]\left(z_{0}\right) \cdot \vartheta\left[\begin{array}{c}
a_{2} \\
b_{2}+a_{1}
\end{array}\right]\left(z_{2}\right)
$$

Since $\vartheta[\mathfrak{m}](z)$ vanishes for the odd characteristic $\left(\begin{array}{l}1 \\ 1\end{array}\right)$, we get that $\vartheta\left[\begin{array}{l}11 \\ 00\end{array}\right](2 Z)$ vanishes along $2 z_{1}=1$.

Next we restrict $\vartheta\left[\begin{array}{l}10 \\ 00\end{array}\right](Z)$ to $2 z_{1}=1$. The result is

$$
\sum \mathrm{e}^{\pi \mathrm{i}\left(z_{0}\left(g_{1}+1 / 2\right)^{2}+\left(g_{1}+1 / 2\right) g_{2}+z_{2} g_{2}^{2}\right)}
$$

we replace $g_{1}$ by $-g_{1}-1$. Then we use that

$$
\left(-g_{1}-1 / 2\right) g_{2} \equiv\left(g_{1}+1 / 2\right) g_{2}+g_{2} \bmod 2
$$

This shows that $\vartheta\left[\begin{array}{l}10 \\ 00\end{array}\right](Z)$ and $\vartheta\left[\begin{array}{l}10 \\ 01\end{array}\right](Z)$ agree on $2 z_{1}=1$. This settles $D_{1}^{-}$.

Next we treat $D_{1}^{+}$: We use the unimodular transformation $Z \mapsto Z\left[\begin{array}{l}10 \\ 21\end{array}\right]$. This transforms the set $2 z_{1}+4 z_{2}=1$ to the set $2 z_{1}=1$. The form $\vartheta\left[\begin{array}{l}11 \\ 00\end{array}\right]$ $(2 Z)$ is invariant under this transformation. Hence it vanishes also on $2 z_{1}+$ $4 z_{2}=1$. Similarly $\vartheta\left[\begin{array}{l}10 \\ 00\end{array}\right](Z)$ is invariant, but $\vartheta\left[\begin{array}{l}10 \\ 01\end{array}\right](Z)$ changes its sign. Hence $D_{1}^{+}$has been reduced to $D_{1}^{-}$.

Next we treat $D_{2}^{-}$. We use the transformation $Z \mapsto Z\left[\begin{array}{l}11 \\ 01\end{array}\right]$. It transforms the set $z_{0}+z_{1}=1$ to the set $z_{1}=1$ and $\vartheta\left[\begin{array}{l}01 \\ 00\end{array}\right](Z)^{2}$ is transformed to 
$\vartheta\left[\begin{array}{l}11 \\ 00\end{array}\right](Z)^{2}$. We have already seen that this vanishes along $z_{1}=1$. The series

$$
\vartheta\left[\begin{array}{l}
00 \\
00
\end{array}\right](Z), \quad \vartheta\left[\begin{array}{l}
10 \\
00
\end{array}\right](Z), \quad \vartheta\left[\begin{array}{l}
00 \\
01
\end{array}\right](Z), \quad \vartheta\left[\begin{array}{l}
10 \\
01
\end{array}\right](Z)
$$

are invariant under the transformation. For the restriction to $z_{1}=1$ we use that $\vartheta\left[\begin{array}{l}a_{1} a_{2} \\ b_{1} b_{2}\end{array}\right](Z)$ goes (see above) to

$$
\mathrm{e}^{\pi \mathrm{i} a_{1} a_{2} / 2} \vartheta\left[\begin{array}{c}
a_{1} \\
b_{1}+a_{2}
\end{array}\right]\left(z_{0}\right) \vartheta\left[\begin{array}{c}
a_{2} \\
b_{2}+a_{1}
\end{array}\right]\left(z_{2}\right)
$$

Hence

$$
\vartheta\left[\begin{array}{c}
00 \\
00
\end{array}\right](Z) \vartheta\left[\begin{array}{c}
10 \\
00
\end{array}\right](Z)-\vartheta\left[\begin{array}{l}
00 \\
01
\end{array}\right](Z) \vartheta\left[\begin{array}{l}
10 \\
01
\end{array}\right](Z)
$$

goes to

$\vartheta\left[\begin{array}{l}0 \\ 0\end{array}\right]\left(z_{0}\right) \vartheta\left[\begin{array}{l}0 \\ 0\end{array}\right]\left(z_{2}\right) \vartheta\left[\begin{array}{l}1 \\ 0\end{array}\right]\left(z_{0}\right) \vartheta\left[\begin{array}{l}0 \\ 1\end{array}\right]\left(z_{2}\right)-\vartheta\left[\begin{array}{l}0 \\ 0\end{array}\right]\left(z_{0}\right) \vartheta\left[\begin{array}{l}0 \\ 1\end{array}\right]\left(z_{2}\right) \vartheta\left[\begin{array}{l}1 \\ 0\end{array}\right]\left(z_{0}\right) \vartheta\left[\begin{array}{l}0 \\ 2\end{array}\right]\left(z_{2}\right)$

which is zero. This settles $D_{2}^{-}$.

To treat $D_{2}^{+}$we use the transformation $Z \mapsto Z\left[\begin{array}{cc}1 & 0 \\ 2 & -1\end{array}\right]$. It transforms the set $z_{0}+z_{1}=1$ to the set $z_{0}+3 z_{1}+2 z_{2}=1$. It leaves $\vartheta\left[\begin{array}{l}01 \\ 00\end{array}\right](Z)^{2}$ invariant. Hence this function vanishes along $z_{0}+3 z_{1}+2 z_{1}=1$. We have seen already that it changes the sign of $\vartheta\left[\begin{array}{l}10 \\ 01\end{array}\right](Z)$. This shows the transformation of the expression $\vartheta\left[\begin{array}{l}00 \\ 00\end{array}\right](Z) \vartheta\left[\begin{array}{l}10 \\ 00\end{array}\right](Z)-\vartheta\left[\begin{array}{l}00 \\ 01\end{array}\right](Z) \vartheta\left[\begin{array}{l}10 \\ 01\end{array}\right](Z)$ just causes a change of the minus sign to a plus sign. Now $D_{2}^{ \pm}$is settled.

We treat $D_{3}^{-}$. First one sees that the transformation $Z \mapsto Z\left[\begin{array}{l}01 \\ 10\end{array}\right]$ interchanges $\vartheta\left[\begin{array}{l}10 \\ 00\end{array}\right](2 Z)$ and $\vartheta\left[\begin{array}{l}01 \\ 00\end{array}\right](2 Z)$. Since this transformation is the identity on $z_{0}=z_{2}$, the difference vanishes on this set.

We still have to investigate the four series

$$
\begin{aligned}
& A: \quad \vartheta\left[\begin{array}{l}
10 \\
00
\end{array}\right](Z), \quad B: \quad \vartheta\left[\begin{array}{l}
00 \\
10
\end{array}\right](Z / 2), \\
& C: \quad \vartheta\left[\begin{array}{l}
00 \\
01
\end{array}\right](Z), \quad D: \quad \vartheta\left[\begin{array}{l}
10 \\
01
\end{array}\right](Z) \text {. }
\end{aligned}
$$

The claim is that $A B=C D$ on $z_{0}=z_{2}$. We use the transformation $Z \mapsto$ $Z\left[\begin{array}{ll}1 & 1 \\ 1 & -1\end{array}\right]$. Then the set $z_{0}=z_{2}$ transforms to $z_{1}=0$. We will use the formula

$$
Z\left[\left(\begin{array}{cc}
1 & 1 \\
1 & -1
\end{array}\right)\left(\begin{array}{l}
g_{1} \\
g_{2}
\end{array}\right)\right]=z_{0}\left(g_{1}+g_{2}\right)^{2}+z_{2}\left(g_{1}-g_{2}\right)^{2} \quad \text { for } \quad z_{1}=0 .
$$

If $g_{1}, g_{2}$ runs through all integers than $h_{1}=g_{1}+g_{2}, h_{2}=g_{1}-g_{2}$ runs through all pairs of integers such that $h_{1}+h_{2}$ is even. For $A$ we get the 
expression

$$
\sum_{h_{1}+h_{2} \text { even }} \mathrm{e}^{\pi \mathrm{i}\left(z_{0}\left(h_{1}+1 / 2\right)^{2}+z_{2}\left(h_{2}+1 / 2\right)^{2}\right)} .
$$

We divide this sum into two parts where $h_{1}, h_{2}$ both are even or both are odd. Both partial sum are equal and we get

$$
A: \quad 2 \vartheta\left[\begin{array}{c}
1 / 2 \\
0
\end{array}\right]\left(4 z_{0}\right) \vartheta\left[\begin{array}{c}
1 / 2 \\
0
\end{array}\right]\left(4 z_{2}\right) \text {. }
$$

In a similar way we split $B, C, D$. The result is

$$
\begin{array}{ll}
B: & \vartheta\left[\begin{array}{l}
0 \\
1
\end{array}\right]\left(2 z_{0}\right) \vartheta\left[\begin{array}{l}
0 \\
1
\end{array}\right]\left(2 z_{2}\right), \\
C: & \vartheta\left[\begin{array}{l}
0 \\
1
\end{array}\right]\left(4 z_{0}\right) \vartheta\left[\begin{array}{l}
0 \\
1
\end{array}\right]\left(4 z_{2}\right) \\
D: & 2 \mathrm{e}^{-\pi \mathrm{i} / 4} \vartheta\left[\begin{array}{c}
1 / 2 \\
1
\end{array}\right]\left(4 z_{0}\right) \vartheta\left[\begin{array}{c}
1 / 2 \\
1
\end{array}\right]\left(4 z_{2}\right) .
\end{array}
$$

The relation $A B=C D$ means

$$
\vartheta\left[\begin{array}{c}
1 / 2 \\
0
\end{array}\right](2 z) \vartheta\left[\begin{array}{l}
0 \\
1
\end{array}\right](z)=\mathrm{e}^{-\pi \mathrm{i} / 4} \vartheta\left[\begin{array}{l}
0 \\
1
\end{array}\right](2 z) \vartheta\left[\begin{array}{c}
1 / 2 \\
1
\end{array}\right](2 z) .
$$

This relation between theta series of one variable is left as an exercise.

The case $D_{3}^{+}$is similar.

Lemma 5.4. The divisors $D_{i}^{-}$correspond (close to the standard node) to divisors of the form $H(S, d)$ :

$$
\begin{array}{ll}
D_{1}^{-}: & S=1 / 4\left(\begin{array}{cc}
0 & 1 \\
1 & 0
\end{array}\right), \quad d=\frac{1}{4}, \\
D_{2}^{-}: & S=1 / 4\left(\begin{array}{cc}
2 & 1 \\
1 & 0
\end{array}\right), \quad d=\frac{1}{2}, \\
D_{3}^{-}: & S=1 / 4\left(\begin{array}{cc}
1 & 0 \\
0 & -1
\end{array}\right), \quad d=0 .
\end{array}
$$

Notice that $S$ is a primitive element of $\mathcal{T}^{*}$. We compute the value $J(U, Z)$ of the cocycle of the associated local Borcherds product for some $U$. Recall (4.5) that

$$
J(U, Z):=B(Z[U]) / B(Z)=C_{U} \mathrm{e}^{2 \pi \mathrm{i} \sigma\left(Z H_{U}\right)} .
$$

The constant $C_{U}$ is not important, since it is a root of unity that disappears if one takes a suitable power. Hence we only give our attention to $H_{U}$. We 
compute them for the generators given in Lemma 5.1. Evaluation of the formula given in Lemma 4.5 gives the following values for the $8 H_{V_{i}}$ :

$$
\begin{aligned}
& D_{1}^{-}: \quad\left(\begin{array}{ll}
0 & 0 \\
0 & 0
\end{array}\right), \quad\left(\begin{array}{cc}
-4 & -2 \\
-2 & 0
\end{array}\right), \quad\left(\begin{array}{cc}
0 & -2 \\
-2 & -8
\end{array}\right), \quad\left(\begin{array}{ll}
-12 & -18 \\
-18 & -24
\end{array}\right) \text {, } \\
& D_{2}^{-}: \quad\left(\begin{array}{ll}
0 & 0 \\
0 & 0
\end{array}\right), \quad\left(\begin{array}{cc}
-4 & -2 \\
-2 & 0
\end{array}\right), \quad\left(\begin{array}{ll}
0 & 0 \\
0 & 0
\end{array}\right), \quad\left(\begin{array}{ll}
0 & 0 \\
0 & 0
\end{array}\right) \text {. } \\
& D_{3}^{-}: \quad\left(\begin{array}{ll}
0 & 0 \\
0 & 0
\end{array}\right), \quad\left(\begin{array}{cc}
-4 & -2 \\
-2 & 0
\end{array}\right), \quad\left(\begin{array}{ll}
0 & 0 \\
0 & 0
\end{array}\right), \quad\left(\begin{array}{ll}
0 & 0 \\
0 & 0
\end{array}\right) \text {. }
\end{aligned}
$$

To compare them we make use of trivial divisors $\mathrm{e}^{\sigma\left(U Z^{\mathrm{t}} U\right)} / \mathrm{e}^{\sigma(Z)}$. For $U=V_{1}$ and $U=V_{2}$ one gets the following values:

$$
\begin{aligned}
& \left(V_{1}\right): \quad\left(\begin{array}{ll}
0 & 0 \\
0 & 0
\end{array}\right), \quad\left(\begin{array}{ll}
0 & 0 \\
0 & 0
\end{array}\right), \quad\left(\begin{array}{cc}
0 & 4 \\
4 & 16
\end{array}\right), \quad\left(\begin{array}{cc}
8 & 12 \\
12 & 16
\end{array}\right), \\
& \left(V_{2}\right): \quad\left(\begin{array}{ll}
0 & 0 \\
0 & 0
\end{array}\right), \quad\left(\begin{array}{ll}
4 & 2 \\
2 & 0
\end{array}\right), \quad\left(\begin{array}{ll}
0 & 0 \\
0 & 0
\end{array}\right), \quad\left(\begin{array}{ll}
4 & 6 \\
6 & 8
\end{array}\right) \text {. }
\end{aligned}
$$

From these data the following proposition follows.

Proposition 5.1. Consider the divisors $D_{1}^{ \pm}, D_{2}^{ \pm}, D_{3}^{ \pm}$. Their classes in $\mathrm{Cl}(\mathcal{X}, \eta)$ are not zero and satisfy the relations $D_{i}^{+}=-D_{i}^{-}$and

$$
D_{1}^{ \pm}=-D_{2}^{ \pm}=-D_{3}^{ \pm}
$$

Remark. The proof of Proposition 5.1 was long and tedious and used the modular approach. In this context the node appeared as a zero-dimensional cusp of a Siegel modular variety. A theory of local Borcherds products opened us a way to study the local divisor class group of such a cusp. Since a node is only a very extreme example of a cusp one may ask whether this is an adequate method. So one can ask whether there is a direct proof of Proposition 5.1. This is actually the case as a referee of the paper explained to us. He brought our attention to the fact that the node is an isolated intersection point of $D_{1}^{+}$and $D_{2}^{-}$. This can be checked easily with the help of a computer. One also can check that both divisors are smooth at the node. This is enough to show that $D_{1}^{+}$and $D_{2}^{-}$agree in $\operatorname{Cl}(\mathcal{X}, \eta)$. For sake of completeness we give the argument. It has been explained to us by Slawomir Cynk. It uses the blow up (big resolution) of the node. Recall that the exceptional divisor of the blow up of the node is isomorphic to $\mathbb{P}^{1} \times \mathbb{P}^{1}$. We use the following fact. A divisor (in a neighborhood of the node) is a Cartier divisor (in a small neighborhood of the node) if and only if the intersection 
number of its strict transform (in the big resolution) with a horizontal and a vertical line in $\mathbb{P}^{1} \times \mathbb{P}^{1}$ is the same.

The strict transform of a smooth surface as $D_{1}^{+}$intersects $\mathbb{P}^{1} \times \mathbb{P}^{1}$ in line $\ell$. By the fact mentioned above, $\ell$ must be a horizontal line or a vertical line. Since $D_{1}^{+}$and $D_{2}^{-}$have the node as isolated fixed point, the two corresponding lines must be both horizontal or both vertical. But then $D_{1}^{+}-D_{2}^{-}$ is a Cartier divisor (again using the mentioned fact above). The same argument works for $D_{1}^{+}$and $D_{3}^{-}$. This gives a new proof of Proposition 5.1.

The stabilizer of a node $a$ acts on the local Picard group $\mathrm{Cl}(\mathcal{X}, a)$. The subgroup of index two that fixes the rulings acts as identity. Hence we see the following result.

Remark 5.1. By means of Proposition 5.1 and Theorem 1.1 it is possible to compute the image of each of the 188 basic divisors in $\mathrm{Cl}(\mathcal{X}, \eta)$. Since they generate $\mathrm{Cl}(\mathcal{X}) \otimes_{\mathbb{Z}} \mathbb{Q}$, we have a complete description of the map

$$
\mathrm{Cl}(\mathcal{X}) \otimes_{\mathbb{Z}} \mathbb{Q} \longrightarrow \mathrm{Cl}(\mathcal{X}, \eta) \otimes_{\mathbb{Z}} \mathbb{Q} \quad(\cong \mathbb{Q}) .
$$

Hyperplane sections of $\mathcal{X}$ define line bundles and hence are trivial at any point. Hence the one-dimensional representation is in the kernel of the map

$$
\mathrm{Cl}(\mathcal{X}) \otimes_{\mathbb{Z}} \mathbb{Q} \longrightarrow \bigoplus_{a \text { node }} \mathrm{Cl}(\mathcal{X}, a) \otimes_{\mathbb{Z}} \mathbb{Q}
$$

From the above explicit description one can compute that the onedimensional representation is the precise kernel. This might be a general phenomenon for Siegel three-folds (compare [1, Theorem 5.4]).

\section{Projective resolutions}

If $D$ is an effective divisor on an irreducible normal complex space $X$, then we associate the ideal sheaf $\mathcal{I}(D)$ of all holomorphic functions on open subsets that satisfy $(f) \geq D$ on this subset. The blow up of $D$, by definition, is the blow up of the ideal sheaf $\mathcal{I}(D)$. If $\mathcal{I}(D)$ is invertible (i.e., locally the divisor of a holomorphic function) then the blow up changes nothing. More generally, suppose that $\mathcal{J}$ is an invertible ideal sheaf then $\mathcal{I}(D)$ and $\mathcal{I}(D) \mathcal{J}$ have the same blow up. It follows that the blow up of an effective divisor depends only on its image in the divisor class group $\mathrm{Cl}(X)$.

The relation $\mathcal{I}(D)^{n}=\mathcal{I}(n D)$ is not true in general. But in our situation it is true. 
Lemma 6.1. Let $Q$ be the affine quadric defined by $z_{1} z_{4}=z_{2} z_{3}$ and let $P$ be the prim divisor defined by $z_{1}=z_{2}=0$. Then

$$
\mathcal{I}(n P)=\mathcal{I}(P)^{n}
$$

Proof. We have to show $\mathcal{I}(n P) \subset \mathcal{I}(P)^{n}$ for $n>0$ since the converse inclusion is trivial. Hence we have to consider an element $f$ of the (analytic) local ring $\mathcal{O}_{Q, 0}$ that vanishes along $P$ of order at least $n$. We represent it by a power series $F$. Replacing terms $z_{2} z_{3}$ by $z_{1} z_{4}$ we can manage that it is of the form

$$
\begin{aligned}
F\left(z_{1}, z_{2}, z_{3}, z_{4}\right) & =F_{1}\left(z_{1}, z_{2}, z_{4}\right)+z_{3} F_{2}\left(z_{1}, z_{3}, z_{4}\right) \\
& =\sum a_{\nu_{1}, \nu_{2}, \nu_{4}} z_{1}^{\nu_{1}} z_{2}^{\nu_{2}} z_{4}^{\nu_{4}}+\sum_{\nu_{3}>0} b_{\nu_{1}, \nu_{2}, \nu_{4}} z_{1}^{\nu_{1}} z_{3}^{\nu_{3}} z_{4}^{\nu_{4}}
\end{aligned}
$$

We test the vanishing of $f$ along $z_{1}=z_{2}=0$ of order $\geq n$ in the chart $z_{3} \neq 0$. Here we have

$$
\begin{aligned}
f & =F_{1}\left(z_{1}, z_{1} z_{4} / z_{3}, z_{4}\right)+z_{3} F_{2}\left(z_{1}, z_{3}, z_{4}\right) \\
& =\sum a_{\nu_{1}, \nu_{2}, \nu_{4}} z_{1}^{\nu_{1}+\nu_{2}} z_{3}^{-\nu_{2}} z_{4}^{\nu_{2}+\nu_{4}}+\sum_{\nu_{1}>0} b_{\nu_{1}, \nu_{3}, \nu_{4}} z_{1}^{\nu_{1}} z_{3}^{\nu_{3}} z_{4}^{\nu_{4}} .
\end{aligned}
$$

The divisor is given in this chart by one equation $z_{1}=0$. The vanishing of order $\geq n$ along this divisor implies

$$
a_{\nu_{1}, \nu_{2}, \nu_{3}} \neq 0 \Longrightarrow \nu_{1}+\nu_{2} \geq n, \quad b_{\nu_{1}, \nu_{2}, \nu_{3}} \neq 0 \Longrightarrow \nu_{1} \geq n
$$

This shows that $F$ is contained in the ideal generated by $z_{1}^{\nu_{1}} z_{2}^{\nu_{2}}, \nu_{1}+\nu_{2} \geq n$, and hence $f \in \mathcal{I}(P)^{n}$.

Let $(X, a)$ be a three-dimensional nodal singularity. The blow up of a divisor $D$ gives a small resolution of the node if and only if $D$ is not trivial at the node. This follows easily from Lemma 6.1 in connection with the structure theorem $\mathrm{Cl}(X, a) \cong \mathbb{Z}$.

We formulate one of the main results of this paper. The group $\overline{\mathcal{H}}$ has been defined in the introduction. Let now $G \subset \overline{\mathcal{H}}$ be $s$ subgroup. We recall that we defined in the introduction two sets $\mathcal{A}, \mathcal{B}$ of nodes depending on $G$. We reformulate the theorem at the end of the introduction in the following more precise form. 
Theorem 6.1. Let $G \subset \overline{\mathcal{H}}$ be a subgroup such that $\mathcal{A} \cup \mathcal{B}$ is the set of all nodes. Assume that for each node $a \in \mathcal{B}, a \notin \mathcal{A}$, the map

$$
\left(\mathrm{Cl}(\mathcal{X}) \otimes_{\mathbb{Z}} \mathbb{Q}\right)^{G} \longrightarrow \mathrm{Cl}(\mathcal{X}, a) \otimes_{\mathbb{Z}} \mathbb{Q}
$$

is not the zero map. Then there exists a projective resolution $\tilde{\mathcal{X}} \rightarrow \mathcal{X}$ such that the action of $G$ extends to a group of biholomorphic transformations of $\tilde{\mathcal{X}}$ and such that $G-\operatorname{Hilb}(\tilde{\mathcal{X}})$ is a resolution of $\mathcal{X} / G$ in the form of a projective Calabi-Yau manifold.

Supplement. For each given $G$, the map in this theorem is computable.

Proof. First we blow up the nodes from $\mathcal{A}$ to get a partial resolution $\mathcal{X}_{1} \rightarrow \mathcal{X}$. The group $G$ extends since the set $\mathcal{A}$ is $G$-invariant. If $\mathcal{X}_{1}$ is smooth we are done. Otherwise we choose some node $a \in \mathcal{X}$ that remains singular in $\mathcal{X}_{1}$. By assumption there exists a divisor $D$ such that its class in $\mathrm{Cl}(\mathcal{X}) \otimes_{\mathbb{Z}} \mathbb{Q}$ is $G$-invariant and that is not trivial at $a$. We can assume that $D$ is effective, since we can add to $D$ the divisor of a homogenous polynomial in $Y_{0}, \ldots, X_{3}$. We can also assume that $D$ itself (and not only its class) is $G$-invariant, since we can replace $G$ by the sum of all $g(D), g \in G$. Now we want to blow up $D$. To be precise, we take the transform $D_{1}$ of $D$ in $\mathcal{X}_{1}$, and blow up $D_{1}$ to produce a partial resolution $\mathcal{X}_{2} \rightarrow \mathcal{X}_{1}$. Of course, the blow up does not change anything in the smooth part of $\mathcal{X}_{1}$ because there any divisor is locally principal. The resolution $\mathcal{X}_{2} \rightarrow \mathcal{X}_{1}$ is a small resolution of certain nodes including the node $a$. If $\mathcal{X}_{2}$ is already smooth we are done. Otherwise we proceed in the same manner.

The constructed $\tilde{\mathcal{X}}$ has the property that for each point of the quotient $\tilde{\mathcal{X}} / G$ there exists a small (analytic) neighborhood $U$ and a holomorphic three-form without zeros on the regular locus of $U$. For the nodes $a \in \mathcal{A}$ one has to observe the following. The Calabi-Yau form gets a zero of order one along the exceptional divisor of the blow up. But, by the definition of $\mathcal{A}$, there is an (central) element $g \in G_{a}$ that fixes the exceptional divisor pointwise. Due to the ramification, the Calabi-Yau form, considered on the quotient of the blow up by $g$, does not vanish along the image of the exceptional divisor.

As a consequence, all singularities of $\tilde{\mathcal{X}} / G$ are of the form $\mathbb{C}^{3} / H$, where $H \subset \mathrm{SL}(3, \mathbb{C})$, is a finite group. But then the results of [2] show that the $G$-Hilbert scheme $G$-Hilb $(\tilde{\mathcal{X}})$ gives a projective resolution in the form of a Calabi-Yau manifold (compare [9, Theorem 2.6]). 


\section{Examples}

(1) It can happen that a group $G \subset \overline{\mathcal{H}}$ contains the conjugates of the transformation $\left(Y_{0},-Y_{1}, Y_{2},-Y_{3}, X_{0}, X_{1},-X_{2},-X_{3}\right)$. For such a group all nodes are contained in $\mathcal{A}$. Hence $\mathcal{X} / G$ admits a projective Calabi-Yau resolution. These examples has been described in detail in [10] (see Lemma 8.1, Remark 8.3 and Corollary 8.4).

(2) We start with a counter-example. There exist groups $G \subset \mathcal{G}$ of order 32 that acts freely on $\mathcal{X}$. An example is the group generated by the following transformations:

$$
\begin{aligned}
& \left(-Y_{1},-\mathrm{i} Y_{0}, Y_{3},-\mathrm{i} Y_{2},-\mathrm{i} X_{1}, X_{0}, \mathrm{i} X_{3}, X_{2}\right), \\
& \left(-\mathrm{i} Y_{2}, Y_{3}, Y_{0}, \mathrm{i} Y_{1}, X_{3},-X_{2},-\mathrm{i} X_{1},-\mathrm{i} X_{0}\right), \\
& \left(-Y_{0}, Y_{1}, Y_{2},-Y_{3}, X_{0},-X_{1}, X_{2},-X_{3}\right), \\
& \left(\mathrm{i} Y_{0}, \mathrm{i} Y_{1}, \mathrm{i} Y_{2}, \mathrm{i} Y_{3},-\mathrm{i} X_{0},-\mathrm{i} X_{1},-\mathrm{i} X_{2},-\mathrm{i} X_{3}\right), \\
& \left(Y_{0}, Y_{1},-Y_{2},-Y_{3}, X_{0}, X_{1},-X_{2},-X_{3}\right), \\
& \left(\mathrm{i} Y_{0}, \mathrm{i} Y_{1}, \mathrm{i} Y_{2}, \mathrm{i} Y_{3}, \mathrm{i} X_{0}, \mathrm{i} X_{1}, \mathrm{i} X_{2}, \mathrm{i} X_{3}\right) .
\end{aligned}
$$

In this case all nodes are of type $\mathcal{B}$, there are none of type $\mathcal{A}$. But the space $\left(\mathrm{Cl}(\mathcal{X}) \otimes_{\mathbb{Z}} \mathbb{Q}\right)^{G}$ is one dimensional (generated by a hyperplane section). The image in $\operatorname{Cl}(\mathcal{X}, a) \otimes_{\mathbb{Z}} \mathbb{Q}$ is zero for all nodes $a$. We claim even more. There is no projective Calabi-Yau manifold $M$ that is birational equivalent to $\mathcal{X} / G$. This follows from a theorem of Kollár that states that two bimeromorphic equivalent models are related by flops ([12, Theorem 4.9]). Hence $M$ must be obtained from $\mathcal{X} / G$ by a small resolution. The pull back to $\mathcal{X}$ would give a projective $G$-invariant resolution. It is easy to show that such a resolution is the blow-up along a $G$-invariant divisor. ${ }^{2}$

Using a calculator we got the following result.

Theorem 7.1. There are 54 conjugacy classes of subgroups $G \subset \overline{\mathcal{H}}$ that act freely on $\mathcal{X}$. Their orders are in $\{1,2,4,8,16,32\}$. For those of order 32 there is no projective Calabi-Yau model for $\mathcal{X} / G$ (but a weak Calabi-Yau model). Those of order 1, 2 and 4 all admit projective Calabi-Yau models. In the case of order 8, 13 cases have a projective $C$ - $Y$ model and 7 cases do not have it. There is one class of order 16 with a projective Calabi-Yau model, the other 12 classes do not have it.

\footnotetext{
${ }^{2}$ We are very grateful to van Geemen who pointed out to us that there exists no projective model for this group $G$.
} 
The quotient of a rigid projective manifold $M$ by a finite freely acting group is rigid. The Euler number is $e(M / G)=e(M) / \# G$. Hence we get rigid Calabi-Yau manifolds with Euler numbers 4, 8, 16, 32.

The freely acting group of order 16 with a projective Calabi-Yau model is of special interest.

Theorem 7.2. The group $G$ generated by the two transformations

$$
\begin{aligned}
& \left(-Y_{2}, \mathrm{i} Y_{3}, \mathrm{i} Y_{0}, Y_{1},-\mathrm{i} X_{3},-\mathrm{i} X_{2}, X_{1},-X_{0}\right), \\
& \left(\mathrm{i} Y_{1}, Y_{0},-\mathrm{i} Y_{3}, Y_{2}, X_{1}, \mathrm{i} X_{0}, X_{3},-\mathrm{i} X_{2}\right)
\end{aligned}
$$

has order 16 and acts freely on $\mathcal{X}$. The quotient $\mathcal{X} / G$ has a resolution in the form of a rigid Calabi-Yau manifold $\left(h^{12}=0\right)$ with Euler number $e=4$ and Picard number $h^{11}=2$.

(3) We consider the group $G$ of order 2 that is generated by the involution

$$
\sigma_{1}: \quad\left(Y_{0}, Y_{1}, Y_{2}, Y_{3},-X_{0},-X_{1},-X_{1},-X_{3}\right) \text {. }
$$

It generates a normal subgroup $G$ of $\overline{\mathcal{G}}$. The involution is fixed point free. Hence all nodes are of type $\mathcal{B}$ (and there are no ones of type $\mathcal{A}$ ). The divisor $D_{2}^{+}\left(X_{0} X_{2}+X_{1} X_{3}=Y_{0} Y_{1} \pm Y_{2} Y_{3}=0\right)$ is invariant under $G$ and it is not trivial at the standard node (see Proposition 5.1). Since $G$ is normal we obtain by transformation for each node a divisor that is $G$-invariant and not trivial at this node. Hence we get a projective model.

(4) Next, we consider the group of order 2 that is generated by the involution

$$
\sigma_{2}:=\left(Y_{0},-Y_{1},-Y_{2}, Y_{3}, X_{0},-X_{1},-X_{2}, X_{3}\right) .
$$

This substitution has six conjugates in $\overline{\mathcal{H}}$, namely

$$
\begin{aligned}
& \left(Y_{0},-Y_{1}, Y_{2},-Y_{3},-X_{0},-X_{1}, X_{2}, X_{3}\right), \\
& \left(Y_{0},-Y_{1},-Y_{2}, Y_{3}, X_{0},-X_{1},-X_{2}, X_{3}\right), \\
& \left(Y_{0}, Y_{1},-Y_{2},-Y_{3},-X_{0}, X_{1},-X_{2}, X_{3}\right), \\
& \left(Y_{0},-Y_{1},-Y_{2}, Y_{3},-X_{0}, X_{1}, X_{2},-X_{3}\right), \\
& \left(Y_{0}, Y_{1},-Y_{2},-Y_{3}, X_{0},-X_{1}, X_{2},-X_{3}\right), \\
& \left(Y_{0},-Y_{1}, Y_{2},-Y_{3}, X_{0}, X_{1},-X_{2},-X_{3}\right) .
\end{aligned}
$$

Again the divisor $D_{2}^{+}$is invariant under them and not trivial at the standard node. So we get projectivity again. 
There are 10 conjugacy classes of involutions in $\overline{\mathcal{H}}$. In [10] we listed them in Proposition 7.6 and computed the divisor class and Euler numbers for them. As in the case $\sigma_{1}, \sigma_{2}$ above one can verify in each of the cases that Theorem 6.1 applies to obtain a projective Calabi-Yau manifold again. Then we obtain the following theorem.

Theorem 7.3. The following table describes fixed point sets of the involutions $\sigma_{i}, i \leq i \leq 10$, on $\mathcal{X}$ and the Hodge numbers of a (projective) Calabi-Yau model of the quotient $\mathcal{X} / \sigma_{i}$.

$\begin{array}{llll} & \text { Fixed points } & h^{11} & h^{12} \\ \sigma_{1}: & \text { empty set } & 16 & 0 \\ \sigma_{2}: & 16 \text { nodes } & 40 & 0 \\ \sigma_{3}: & 4 \text { elliptic curves } & 20 & 4 \\ \sigma_{4}: & \text { empty set } & 16 & 0 \\ \sigma_{5}: & \text { empty set } & 16 & 0 \\ \sigma_{6}: & 8 \text { conics in planes }\left(\cong \mathbb{P}^{1}\right) & 28 & 0 \\ \sigma_{7}: & 8 \text { lines }\left(\cong \mathbb{P}^{1}\right) & 28 & 0 \\ \sigma_{8}: & 2 \text { elliptic curves } & 18 & 2 \\ \sigma_{9}: & 2 \text { elliptic curves } & 18 & 2 \\ \sigma_{10}: & 4 \text { conics in planes }\left(\cong \mathbb{P}^{1}\right) & 22 & 0\end{array}$

In the cases where elliptic curves are in the fixed point set, we get non rigid Calabi-Yau manifolds.

(5) Next we consider a group of order 3 .

Proposition 7.1. There is only one conjugacy class of elements of order three in $\overline{\mathcal{H}}$. It can be represented by

$$
\left(Y_{0}, Y_{3}, Y_{1}, Y_{2}, X_{0}, X_{3}, X_{1}, X_{2}\right)
$$

Its fixed point set in $\mathcal{X}$ does not contain a node. It consists of one elliptic curve and four isolated points. The fixed point set in $\tilde{\mathcal{X}}$ is the same. The subgroup of order three leads to a projective Calabi-Yau model with Hodge numbers

$$
\left(h^{11}, h^{12}\right)=(18,2)
$$

Proof (sketch). We omit the computation of the fixed points. We can extend the group to a small resolution $\tilde{\mathcal{X}}$ of $\mathcal{X}$. Now the Euler number can be easily computed by means of the stringy formula (compare [10]). The result 
is $e=32$. Next we compute the Picard number $h^{11}$. The invariant part $\operatorname{dim} \mathrm{Cl}(\mathcal{X})^{G}$ has rank 12 as can be proved by means of the known character of the action of $\overline{\mathcal{H}}$ (see [10]). One can check that over isolated points there is one exceptional divisor but over the elliptic curve there are two. Hence we have six exceptional divisors. We obtain $h^{11}=18$.

\section{A special class of groups}

This section rests highly on computer calculations. We consider all subgroups of $\mathcal{H}$ that are isomorphic to $(\mathbb{Z} / 2 \mathbb{Z})^{m}$. There are 165 conjugacy classes. In all 165 cases the group extends to a small resolution $\mathcal{X}$ such that a weak Calabi-Yau model can be obtained by a resolution of $\mathcal{X} / G$. There are 144 classes that admit a projective Calabi-Yau model that can be obtained as described in the introduction. This means that the group $G$ extends to a not necessarily minimal projective resolution $\tilde{\mathcal{X}}$ such the $\tilde{\mathcal{X}} / G$ admits a resolution in the form of a (projective) Calabi-Yau manifold. It is known that the Euler number and divisor class number are bimeromorphic invariant for weak Calabi-Yau manifolds (since they are related by flops.) Therefore, we always can use a small minimal resolution $\tilde{\mathcal{X}}$ (also a non-projective one) for the computation of the Euler and divisor class number. This actually means that we sometimes compute the Hodge numbers for a group with projective Calabi-Yau model using a non-projective model.

\subsection{Computation of the divisor class number}

So let $G \subset \overline{\mathcal{H}}$ be a subgroup that is isomorphic to $(\mathbb{Z} / 2 \mathbb{Z})^{m}$. We choose a small resolution $\tilde{\mathcal{X}}$ such that $G$ extends. We have to determine the divisor class number of a Calabi-Yau resolution of $\tilde{\mathcal{X}} / G$. This is the sum of the divisor class number of $\mathcal{X} / G$ and the number of exceptional divisors. The divisor class number of $\mathcal{X} / G$ can be computed since we know the representation of $\overline{\mathcal{H}}$ on $\mathrm{Cl}(\mathcal{X}) \otimes_{\mathbb{Z}} \mathbb{Q}$. This can be done by a program. To get the number of exceptional divisors we need information about the singularities of $\mathcal{X} / G$. Locally, they are of the form $\mathbb{C}^{3} / H$ with an abelian group $H \subset \operatorname{SL}(3, \mathbb{Z})$. where all elements of $H$ have order $\leq 2$. After diagonalization $H$ consists of sign changes. Since the determinants are one we have an even number of sign changes. In this cases the resolution is easy to produce (see [9]). The result is that the singular locus consists of one line or of three crossing lines. In the first case we have one exceptional divisor in the resolution in the second case we have three. This shows that the number of exceptional divisors of a (weak) Calabi-Yau resolution of $\tilde{\mathcal{X}} / G$ equals the number of components 
of the fixed point locus. We want to express this in the singular model $\mathcal{X}$. So let $C \cong \mathbb{P}^{1}$ be an exceptional curve that projects to a node in $\mathcal{X}$. Then there is an $g \in G, g \neq e$, that fixes the node. This node must be an isolated fixed point of $g$ since otherwise its fixed locus on $\tilde{\mathcal{X}}$ would be not smooth. (Compare Lemma 8.2 below for a more detailed description.) So we see the following lemma.

Lemma 8.1. Let $G \subset \overline{\mathcal{H}}$ be a subgroup of type $(\mathbb{Z} / 2 \mathbb{Z})^{m}$. Let a be the number of $G$-equivalence classes of irreducible curves in $\mathcal{X}$ that are in the fixed point locus and let $b$ be the number of $G$-equivalence classes of nodes that are isolated fixed points. Then the divisor class number of a weak Calabi-Yau model of $\mathcal{X} / G$ equals

$$
a+b+\operatorname{dim}\left(\operatorname{Cl}(\mathcal{X}) \otimes_{\mathbb{Z}} \mathbb{Q}\right)^{G}
$$

\subsection{Computation of the Euler number}

Our main tool will be the string theoretic formula [14]:

Let $X$ be a weak Calabi-Yau three-fold and $G$ a finite group of biholomorphic transformations that leave the Calabi-Yau three-form invariant. Assume that the stabilizers $G_{a}$ are contained in the special linear group of the tangent space. Then the Euler number of a resolution of $X / G$ in the form of a Calabi-Yau manifold equals

$$
e(M, G)=\frac{1}{\# G} \sum_{g h=h g} e\left(M^{\langle g, h\rangle}\right) .
$$

Here $M^{\langle g, h\rangle}$ denotes the common fixed point set of $g, h$.

Let $G \subset \overline{\mathcal{H}}$ be a subgroup that extends to a small resolution $\tilde{\mathcal{X}}$. Then the string theoretic formula applies. Recall that the fixed point locus of $G$ on $\tilde{\mathcal{X}}$ is a curve $C$ with smooth irreducible components. Let $S$ be the singular locus of $C$ (crossing points). Then $e(C)=e(C-S)+\# S$. The Euler number of $C-S$ is the sum of the Euler numbers of its connected components. The Euler number of a smooth non-compact curve $K$ with compactification $\bar{K}$ is just

$$
e(K)=2-2 g(\bar{K})-\#(\bar{K}-K) \quad(g(\bar{K})=\text { genus }) .
$$

Since we know the equations of $\mathcal{X}$, it is no problem to compute the fixed point loci on $\mathcal{X}$. This is the image of $C$ in $\mathcal{X}$. We know already that the fixed point loci of elements of order two consist of (smooth) rational and elliptic curves and isolated points (nodes). Hence we have just to analyze 
what happens on a exceptional $\mathbb{P}^{1}$ in $\tilde{\mathcal{X}}$. This is clarified by the following two lemmas.

Lemma 8.2. Let $g \in \overline{\mathcal{H}}$ an element of order two which fixes a node a. There are two cases:

(1) $a$ is an isolated fixed point of $g$. In this case $g$ extends as identity on the exceptional $\mathbb{P}^{1}$ over $a$.

(2) a is not an isolated fixed point of $g$. Then $g$ has precisely two fixed points on the exceptional $\mathbb{P}^{1}$. These two fixed points are intersection points of two further fixed point curves of $g$ which are visible already in $\mathcal{X}$.

This lemma follows from our investigations of the involutions during the proof of Theorem 7.3.

Lemma 8.3. Let $g, h$ be two different commuting elements of $\overline{\mathcal{H}}$ of order two. Assume that they fix a joint node a. Then there are two possibilities for the common fixed point locus on the exceptional $\mathbb{P}^{1}$ over a.

(1) It consists of two points. This happens if a is an isolated fixed point of one of the three $\{g, h, g h\}$.

(2) It is empty. This happens if it is not an isolated fixpoint of any of the three $\{g, h, g h\}$.

Proof. We first mention that it cannot happen that $a$ is an isolated fixed point of two of the $g, h, g h$. The reason is that there is only one conjugacy class of elements of order two which fix a node as isolated fixed point (the conjugacy class of $\sigma_{2}$ ). This conjugacy class consists of 6 elements and each of them fixes 16 nodes and nothing else. The 6 blocks of 16 nodes are pairwise disjoint and exhaust all $96=6 \cdot 16$ nodes.

Case (1) is clear, assume for example that $g h$ has the node as an isolated fixed point. Then it acts as identity on the exceptional fiber. Then $g$ and $h$ are inverse on this fiber and have the same fixed points. From Lemma 8.2 we know that they are two.

We treat the second case. From the assumption it follows that $g$ and $h$ are different. We know that $g$ has precisely two fixed points on the exceptional fiber over $a$. We choose the biholomorphic map $\mathbb{P}^{1} \cong \overline{\mathbb{C}}$ such that $g$ acts as $g(z)=-z$. Since $h$ commutes with $g$ it acts on the two fixed points. It cannot fix both since then $h$ would we equal to $g$. Hence $h$ permutes 0 
and $\infty$ and the only possibilities are the transformations $h(z)= \pm 1 / z$. But they have different fixed points. Hence the joint fixed point set on the $\mathbb{P}^{1}$ is empty.

We have collected all what we need for the computation of the Euler number. A computer calculation gives now the following list.

There are 40 different pairs $(\mathrm{cl}, e)$ of divisor class numbers and Euler numbers of weak Calabi-Yau manifolds that are produced by subgroups of $\overline{\mathcal{H}}$ isomorphic to $(\mathbb{Z} / 2 \mathbb{Z})^{m}$ :

$(28,56),(20,40),(16,32),(14,28),(15,26),(12,16),(10,20),(8,16),(4,8)$, $(26,50),(10,8),(22,44),(14,26),(18,28),(13,20),(44,88),(11,16),(16,28)$, $(34,68),(20,32),(16,16),(14,20),(13,26),(22,40),(15,28),(41,82),(26,52)$, $(6,8),(12,8),(17,32),(19,38),(70,140),(14,16),(12,20),(10,16),(9,14)$, $(46,92),(40,80),(32,64),(18,32)$.

There are 33 different pairs $\left(h^{11}, h^{12}\right)$ of Hodge numbers which are produced by subgroups of $\overline{\mathcal{H}}$ isomorphic to $(\mathbb{Z} / 2 \mathbb{Z})^{m}$ and with a projective model as described in the introduction:

$$
\begin{aligned}
& (14,0),(12,4),(20,0),(14,1),(6,2),(26,0),(32,0),(26,1),(70,0),(18,2), \\
& (16,8),(44,0),(15,2),(15,1),(12,2),(10,2),(14,6),(9,2),(4,0),(22,2), \\
& (10,0),(16,0),(14,4),(12,8),(22,0),(20,4),(13,0),(28,0),(19,0),(16,2), \\
& (34,0),(40,0),(8,0) .
\end{aligned}
$$

The list seems to contain several examples that are not contained in the (physicists) literature (see [5]).

\section{Two more examples}

We consider the group $H$ of order 16 that we described in Theorem 7.2. There are transformations of order 3 in $\overline{\mathcal{H}}$ that normalize this group, for example

$$
h_{3}: \quad\left(Y_{0},-Y_{3}, \mathrm{i} Y_{1}, \mathrm{i} Y_{2}, X_{3}, X_{1}, X_{0}, X_{2}\right) .
$$

We consider now the group $H_{48}$ of that is generated by this element and the group in Theorem 7.2. The order of this group is 48. Using Proposition 6.2 on can check that $H_{48}$ extends to a projective small resolution $\tilde{\mathcal{X}} \rightarrow \mathcal{X}$. All 32 elements which are not contained in $H$ are of order 3 . The 16 subgroups of order 3 of $H_{48}$ are conjugated under $H$. This means that the fixed point 
locus of $h_{3}$ in $\mathcal{X}$ maps under $\mathcal{X} \rightarrow \mathcal{X} / H$ biholomorphic to the whole fixed point locus of $H_{48} / H$. Recall the fixed point locus of $h_{3}$ does not contain a node and consists of an elliptic curve and four points. The $H_{48}$-invariant part of $\mathrm{Cl}(\mathcal{X})$ has rank two. Hence the Picard number of a Calabi-Yau resolution of $\mathcal{X} / H_{48}$ equals 8 . The string theoretic formula applied to $\tilde{\mathcal{X}} / H, H_{48} / H$ gives $e=(1 / 3)(4+8 \cdot 4)=12$. Hence we obtain a Calabi-Yau manifold with Hodge numbers

$$
H_{48}: \quad h^{11}=8, \quad h^{12}=2 \quad(e=12) .
$$

Our last example starts with the same group $H$ but we extend it now by an involution

$$
h_{2}: \quad\left(Y_{2}, \mathrm{i} Y_{3},-\mathrm{i} Y_{0},-Y_{1},-\mathrm{i} X_{3}, \mathrm{i} X_{2},-X_{1}, X_{0}\right)
$$

This involution normalizes $H$. Hence we get a group $H_{32}$ of order 32 . Using Proposition 6.2 on can check that $H_{32}$ extends to a projective small resolution $\tilde{\mathcal{X}} \rightarrow \mathcal{X}$. The whole fixed point locus of $H_{32}$ consists of 24 elliptic curves and 32 rational curves. The image in $\mathcal{X} / H$ (i.e., the fixed poind locus of $h_{2}$ acting on $\mathcal{X} / H$ consists of three elliptic curves and 2 rational curves. One obtains a (projective) Calabi-Yau manifold with Hodge numbers

$$
H_{32}: \quad h^{11}=7, \quad h^{12}=3 \quad(e=8) .
$$

Final remark. With a refinement of the described methods we could compute in 1344 of the 4117 cases the Euler number where 786 of the 1344 admit a projective Calabi-Yau model. Euler numbers of weak Calabi-Yau models that we got are

$2,4,6,8,10,12,14,16,18,20,22,24,26,28,30,32,34,38,40,44,46,50,52,56$, $58,64,68,70,80,82,88,92,100,140$.

Euler numbers of a projective Calabi-Yau model from this list are

$$
4,8,12,14,16,20,24,26,28,32,38,40,44,50,52,56,64,68,80,88,100,140 .
$$

The Euler numbers, we could get, are all positive. This is clear from the formula for string theoretic Euler number in case that the group extends to a small resolution. So we cannot get mirror pairs. 


\section{Acknowledgments}

We want to thank the referee (who is unknown to us) and Slawomir Cynk for his advice and patient assistance. We also want to thank Bert van Geemen for his help.

\section{Appendix. The computer computations}

We used the computer algebra system MAGMA [3]. The calculations that need a base field have been done over the field $K=\mathbb{Q}(\zeta)$ where $\zeta$ is an eighth root of unity. The group $\mathcal{G}$ that we described in the introduction as subgroup of $\mathrm{GL}(8, \mathbb{C})$ has been implemented in this way as a matrix subgroup of $\mathrm{GL}(8, K)$. The 96 nodes are described by 96 ideals in the ring $R=K\left[Y_{0}, \ldots, X_{3}\right]$ (containing the defining ideal of $\mathcal{X}$ ). This allows to describe the action of $\mathcal{G}$ on the nodes and one can compute several subgroups, for example the stabilizer of a node. In this way we could verify the transitivity of the group $\mathcal{H}$ on the set of nodes.

The basic thing was that we could compute the action of $\mathcal{G}$ on the vector space $\operatorname{Cl}(\mathcal{X}) \otimes_{\mathbb{Z}} \mathbb{Q}$. From van Geemen and Nygaard we knew that its dimension is 32 . To get the action of $\mathcal{G}$ we constructed 32 divisors (given as prime ideals in $R$ ) which give generates of this space. This has been done already in [10]. We recall briefly the construction. It rests on the modular approach where $Y_{0}, \ldots, X_{3}$ appear as theta functions. But at the end the result can be formulated directly. We considered Igusa's modular form $\chi_{35}$ of weight 35 for the full modular group. We found an expression of its square as a polynomial in $Y_{0}, \ldots, X_{3}$. This polynomial defines a divisor on $\mathcal{X}$. Fortunately the polynomial splits as a product of very simply polynomials of degree one and two. Using the facility of MAGMA to perform the primary decomposition of not too complicated ideals, we decomposed the zero divisor of the polynomial inside $\mathcal{X}$. In this way could construct 132 subvarieties of codimension 1 in $\mathcal{X}$ which are irreducible over $K$. The modular form $\chi_{35}$ is not invariant under the Fricke involution. Since we wanted to include in this paper the Fricke involution, we had to apply it to the 132 subvarieties. The union of both sets contains the 188 elements described in Lemma 1.1. They decompose into three orbits under $\mathcal{G}$. All these varieties are given by explicit prime ideals in $R$. The group $\mathcal{G}$ permutes them and this permutation representation can be computed.

The next problem was to prove that the constructed divisors contain 32 linear independent ones and to select them. For this we constructed 32 curves in $\mathcal{X}$ such that the intersection matrix has rank 32 . Of course these 
curves should not contain a node. (One uses Poincarè duality on the regular locus of $\mathcal{X}$.) For the construction of these curves we intersected arbitrary two of the 132 divisors, decomposed them into irreducible components and selected those which do not run into a node. The rank of the intersection matrix between these curves and the divisors turned out to be 32 . In this way we could construct a basis of $\mathrm{Cl}(\mathcal{X}) \otimes_{\mathbb{Z}} \mathbb{Q}$ and we could compute the action of $\mathcal{G}$ as a homomorphism $\mathcal{G} \rightarrow \mathrm{GL}(32, \mathbb{Q})$.

In Theorem 1.1 we described the images of the generating divisors in the local divisor class groups of the nodes. This can now be implemented as a linear map $\mathbb{Q}^{32} \rightarrow \mathbb{Q}^{96}$ which is equivariant under $\mathcal{G}$.

We computed a list of representatives of the conjugacy classes of subgroups of $\mathcal{H}$. If $G$ is one of these groups we can now compute the invariant space $\left(\mathrm{Cl}(\mathcal{X}) \otimes_{\mathbb{Z}} \mathbb{Q}\right)^{G}$. Then we can compute its image in $\mathbb{Q}^{96}$. So we can decide whether there exists an invariant divisor which is not trivial at a given node. Using Theorem 6.1 we obtained the (projective) Calabi-Yau manifolds that we have described Sections 7-9.

\section{References}

[1] J. H. Bruinier and E. Freitag, Local Borcherds products, Ann. Inst. Fourier (Grenoble) 51 (2001), 1-26.

[2] T. Bridgeland, A. King and M. Reid, The McKay correspondence as an equivalence of derived categories, J. Amer. Math. Soc. 14 (2001), 535-554.

[3] W. Bosma, J. Cannon and C. Playoust, The Magma algebra system. I. The user language, J. Symbolic Comput. 24(3-4) (1997), 235-265.

[4] J.H. Bruinier, G. van der Geer, G. Harder and D. Zagier, The 1-2-3 of modular forms, Universitext, Springer, Berlin, Heidelberg, 2008.

[5] P. Candelas and R. Davies, New Calabi-Yau manifolds with small Hodge numbers, Fortschr. Phys. 58(4-5) (2010), 383-466.

[6] S. Cynk, E. Freitag and R. Salvati-Manni, The geometry and arithmetic of a Calabi-Yau Siegel threefold, accepted by Internat. J. Math. 2010, arXiv: 1004.2997.

[7] S. Cynk and C. Meyer, Modular Calabi-Yau threefolds of level eight, Internat. J. Math. 18(3) (2007), 331-347. 
[8] E. Freitag, Siegelsche Modulfunktionen, Grundlehren der mathematischen Wissenschaften, 254, Springer, Berlin Heidelberg, 1983.

[9] E. Freitag and R. Salvati Manni, Some Siegel three-folds with a CalabiYau model, Ann. Scuola Norm. Sup. Pisa Cl. Sci. (5) IX (2010), 833-850, arXiv: 0905.415.

[10] E. Freitag and R. Salvati Manni, Some Siegel threefolds with a CalabiYau model II, accepted by the Kyungpook Math. J. 2010, arXiv.org: 1001.0324.

[11] B. van Geemen and N.O. Nygaard, On the geometry and arithmetic of some Siegel modular three-folds, J. Number Theory 53 (1995), 45-87.

[12] J. Kollár, Flops, Nagoya Math. J. 113 (1989), 15-36.

[13] M. Reid, La correspondence de McKay, Séminaire Bourbaki 1999/2000, Astérisque 276 (2002), 53-72.

[14] S. Roan, Minimal resolutions of Gorenstein orbifolds in dimension three, Topology 35 (1996), 489-508.

[15] G. Trautmann, Ein Endlichkeitssatz in der analytischen Geometrie, Inventiones Math. 8 (1969), 143-174.

Mathematisches Institut

Im Neuenheimer Feld 288

D69120 HEIDELBERG

E-mail address: freitag@mathi.uni-heidelberg.de

Dipartimento di Matematica

Piazzale Aldo Moro, 2

I-00185 Roma, ItALy

E-mail address: salvati@mat.uniroma1.it

ReCeIved June 26, 2011 